\title{
Force, inertia, metric in Newtonian relativity and in general relativity
}

\author{
P.G.L. Porta Mana \\ <piero.mana@ntnu.no>
}

Draft of 16 October 2016; updated 12 January 2020

This note is an exploration of some notions around kinematics, dynamics, matter, inertia, and force in Newtonian- and general-relativistic continuum mechanics. The conceptual pivot of this exploration is this: Neither in Newtonian relativity or in general relativity should there be any distinction between inertial and non-inertial motion, or between forced and unforced motion.

\section{An exploration}

This note is an exploration of some notions around kinematics, dynamics, matter, inertia, and force in Newtonian- and general-relativistic continuum mechanics. In part, I would like to understand in which possible ways the geometrical objects of each theory belong to the kinematics, and which to the dynamics. The conceptual pivot of this exploration is this:

Neither in Newtonian relativity or in general relativity should there be any distinction between inertial and non-inertial motion, or between forced and unforced motion.

Other related points driving this exploration are, in no particular order:

- The desire to bring to light the concept of force hidden in general relativity. This would be pedagogically useful to understand the Newtonian limit. The balance of forces has advantages over the conservation of momentum in Newtonian mechanics, since the latter is frame-dependent.

- To consider inertia as a force, in Newtonian and in general relativity.

- Newtonian thermomechanics can be restated as a theory of statics in some reference frame, provided inertia is included among the forces (Noll 1963; Truesdell 1991 § I.13). This static point of view is even more natural in general relativity, from a four-dimensional point of view. 
- In general relativity, the notion of geodesic is overestimated. Almost no objects of our everyday experience follow geodesics.

- The desire to keep the differential-geometrical, metric-free nature of many electro-thermo-mechanical notions well in sight, without mixing the metric in them. In Newtonian relativity, for example, the notion of mass density \& flux does not require metric structures of any kind. In general relativity this is also true of energy density \& flux.

- The question: can forces exist where there is no mass? Force and momentum in Newtonian mechanics were traditionally associated with bodies. In general relativity momentum is unhinged from mass, and so probably should force. This goes together with the idea that an electromagnetic field can also sustain a force (Page 1958 § 158, pp. 534-535; Ericksen 2007).

I believe that to appreciate the points above is also useful to compare the particular and precise aims of Newtonian relativity with those, somewhat vaguer, of general relativity.

Being an exploration, so far I've been unable to present this note as a linear logical development. So I plead with you to follow me in my ruffled sequence of observations, questions, and possible answers.

\section{Prerequisites}

I will freely use the differential-geometric notions of inner- and outeroriented forms (also called 'straight' and 'twisted'), explained by Burke (1995; 1987 ch. IV; 1983), Bossavit (1991 chs 2, 3; 1998), Hehl et al. (2003 ch. A), and going back to Schouten \& van Dantzig ${ }^{1}$ at the latest.

I will assume some familiarity with the ' $3+1$ ' decomposition of Einstein's equations ${ }^{2}$ and with the basic principles of Newtonian continuum thermomechanics ${ }^{3}$.

\section{Slicings and frames}

We consider the four-dimensional manifold formed by all physical events - spacetime - as a primitive concept. Speaking of these events we usually adopt notions of simultaneity and of identity of location. These notions

1 Schouten et al. 1940. $\quad{ }^{2}$ Smarr et al. 1978; York 1979; Smarr et al. 1980. $\quad 3$ Truesdell 1991; Samohýl et al. 2014; Truesdell et al. 1960. 
are usually determined by physical phenomena; e.g.we perceive as simultaneous such events as send light rays that reach our eyes at the same time. But we could also decree by convention that a set of events is simultaneous or occurs at the same place, as long as such convention has some differentiability.

A choice of simultaneity is determined by a slicing of spacetime, represented by a function $t$, or equivalently by an exact 1 -form $\mathrm{d} t^{4}$. We call each 3-surface with constant $t$ a slice, and its points places at time $t^{5}$. The choice of a slicing is, for the moment, completely arbitrary.

The 'time' $t$ above is a convention: it does not represent the physical time beaten by a periodic phenomenon, a clock. For a small spacetime displacement $V$ of such a clock, the corresponding small lapse in physical time is given in Newtonian relativity by contraction $\boldsymbol{V} \cdot \mathrm{d} t_{\mathrm{a}}$ with a specific 1 -form $\mathrm{d} t_{\mathrm{a}}$, or equivalently by the square root of the contraction with the degenerate quadratic form $\mathrm{d} t_{\mathrm{a}} \otimes \mathrm{d} t_{\mathrm{a}}$; in general relativity the quadratic form given by the metric $\gamma$ is used instead.

The notion of motion also implies a convention about the correspondence of places on slices at different times, so that we can say 'this place today is the same as that place yesterday'. Such a correspondence can be made with a vector field $U$ such that

$$
u \cdot \mathrm{d} t=1 .
$$

The flow of this vector field puts points on different slices into mutual correspondence. It's sometimes called an observer, or a field of observers, or a frame, the term we use here.

A slicing $t$ and a frame $U$ on an $n$-dimensional manifold induce a natural pair of projection operators: $\mathrm{d} t \otimes \boldsymbol{U}$ projects along the frame, and $\mathbf{I}-\mathrm{d} t \otimes U$ onto the slices. With these projections every $m$-vector $v$ can be represented on each $(n-1)$-slice as the pair $(\hat{v}, \bar{v})$ of an $(m-1)$-vector and an $m$-vector

$$
\hat{v}:=v \cdot \mathrm{d} t, \quad \bar{v}:=v-\hat{v} \wedge \boldsymbol{U} .
$$

Every $m$-form $\alpha$ as the pair $(\hat{\alpha}, \bar{\alpha})$ of an $(m-1)$-form and an $m$-form

$$
\hat{\alpha}:=U \cdot \alpha, \quad \bar{\alpha}:=\alpha-\mathrm{d} t \wedge \hat{\alpha} .
$$

All of the projected components have a well-defined meaning on each slice. The wedge-product, exterior differentiation, and contraction of

$\overline{4}$ York 1979. $\quad{ }^{\mathbf{5}}$ Smarr et al. 1978; York 1979; Smarr et al. 1980; Marsden et al. 1994 § 2.4. 
these objects in the $n$-manifold can be rewritten ${ }^{6}$ as pairs of operations on their slice representatives:

$$
\begin{gathered}
(\hat{\alpha}, \bar{\alpha}) \wedge(\hat{\beta}, \bar{\beta})=\left(\hat{\alpha} \wedge \bar{\beta}+(-1)^{m} \bar{\alpha} \wedge \hat{\beta}, \bar{\alpha} \wedge \bar{\beta}\right) \\
\mathrm{d}(\hat{\alpha}, \bar{\alpha})=\left(\mathrm{L}_{U} \bar{\alpha}-\mathrm{d} \hat{\alpha}, \mathrm{d} \bar{\alpha}\right) \\
(\hat{v}, \bar{v}) \cdot(\hat{\alpha}, \bar{\alpha})=(-\bar{v} \cdot \hat{\alpha}-\hat{v} \cdot \bar{\alpha}, \bar{v} \cdot \bar{\alpha}+\hat{v} \cdot \hat{\alpha}) .
\end{gathered}
$$

In the last expression only one term in $-\bar{v} \cdot \hat{\alpha}-\hat{v} \cdot \bar{\alpha}$ is different from zero, depending on the relative orders of the form $\alpha$ and of the vector $v$. If they have the same order, the full term vanishes.

\section{Matter}

Newtonian continuum mechanics is based on the notions of body and mass, traditionally introduced as separate mathematical objects.

Bodies are represented as abstract three-dimensional manifolds, of which we can consider submanifolds, i.e. sub-bodies. These bodies are immersed and move in space with time. This representation is typical in both Newtonian 7 and Lorentzian or general ${ }^{8}$ relativity. This mathematical representation reflects our experience with 'bodies' we deal with in everyday life, for example a tree lulled by the wind and its individual branches, or a leaf dragged by a water stream, or pieces of differently coloured silly putty getting mixed together.

In Newtonian continuum mechanics, mass is a property of a body, represented as a measure on the respective manifold, satisfying a conservation principle. Conservation of mass, however, can be restated as conservation of 'amount of matter', or more precisely of 'amount of substance' (one of the base quantities of the International System of Units $^{9}$ ). In Newtonian mechanics the two are equivalent, mass being proportional to amount of matter, the proportionality being a constant, different from substance to substance. Conservation of amount of matter is sometimes used instead of mass in the thermomechanics of reacting mixtures ${ }^{10}$ for stoichiometric reasons. Conservation of amount of matter is a manifestation of the conservation of baryonic number ${ }^{11}$.

\footnotetext{
6 Hehl et al. 2003 § B.1.4. $\quad{ }^{7}$ Truesdell 1991 §§ I.2-3; Noll 1959; 1973. $\quad{ }^{8}$ Grot et al. 1966a; Carter et al. 1972. $\quad{ }^{\mathbf{9}}$ Iso 2009a; JCGM 2012. $\quad \mathbf{1 0}^{\mathbf{0}}$ e.g. Eckart 1940a (see especially eq. (7), where $m$ can be dropped); Truesdell 1984 Lect. 6 (in eq. (6.1) $\rho$ can be dropped); Samohýl et al. 2014 and refs therein. $\mathbf{1 1}$ cf. Misner et al. $1973 \S 22.2$.
} 
In general-relativistic continuum mechanics we have to speak of conservation of amount of matter, because mass is no longer a constant property of it; not even rest mass, when particles of different types transform into one another.

It makes thus sense to speak directly of conservation of amount of matter, valid in Newtonian relativity and general relativity.

In both Newtonian ${ }^{12}$ and relativistic ${ }^{13}$ continuum mechanics mass is typically introduced intertwined with the metric ${ }^{14}$.

Mass can be considered as a property of matter in relation to gravitoinertial forces, in both Newtonian and general-relativistic mechanics. If we aim to establish a kinematic of matter independently of any kind of forces and metric, we must then relinquish mass and adopt a more general measure of 'amount of matter', or more precisely of 'amount of substance' (one of the base quantities of the International System of Units ${ }^{15}$ ). Conservation of mass is sometimes indeed replaced by conservation of amount of matter in the Newtonian continuum mechanics of reacting mixtures ${ }^{16}$ owing to stoichiometry. We are considering this replacement independently of particle theories, where conservation of amount of matter is a manifestation of the conservation of baryon number ${ }^{17}$. In the following I'll simply say 'conservation of matter' rather than of 'amount of matter'.

It turns out that the notions of body and amount of matter can be introduced at one stroke ${ }^{18}$, as two aspects of the same entity, and without a metric. The idea is analogous to that of charge ${ }^{19}$.

This beautiful representation is by a closed, outer-oriented 3-form $\boldsymbol{M}$, the matter 3-form, satisfying

$$
\mathrm{d} M=0 .
$$

This object has a simple and beautiful geometrical meaning: it can be visualized in spacetime as a set of uninterrupted tubes possessing a definite orientation along them. The tubes are chosen by us: if we select a closed two-dimensional surface on a spacetime slice, where $\boldsymbol{M} \neq 0$, the matter 3-form selects a unique tube with 3-dimensional section

12 Truesdell 1991 §§ I.4; Noll 1959; 1973. 13 Grot et al. 1966a; Rezzolla et al. 2013 ch. 3. 14 Carter et al. 1972 being an exception. 15 Iso 2009a,b; JCGM 2012. 16 e.g. Eckart 1940a (see especially eq. (7), where $m$ can be dropped); Truesdell 1984 Lect. 6 (in eq. (6.1) $\rho$ can be dropped); Samohýl et al. $2014 \S \S 4.1-4.2$ and refs therein. $\quad 17$ cf. Misner et al. 1973 § 22.2. $\mathbf{1 8}$ cf. Carter et al. 1972. ${ }^{\mathbf{1 9}}$ Burke 1983; Bossavit 1998; Hehl et al. 2000; 2003 § B.1. 
containing that 2-surface. This represents our ability to mark a small body of matter - say, by drawing a ' $\bigcirc$ ' sign on an body, or throwing some leaves in flowing water - and then to follow it as it moves and deforms in space as time passes, with respect to some slicing and frame. A closed outer-oriented 3-form is the perfect mathematical representation of this.

Note that in speaking of 'matter' I am here excluding the electromagnetic field, which is often considered as matter in general

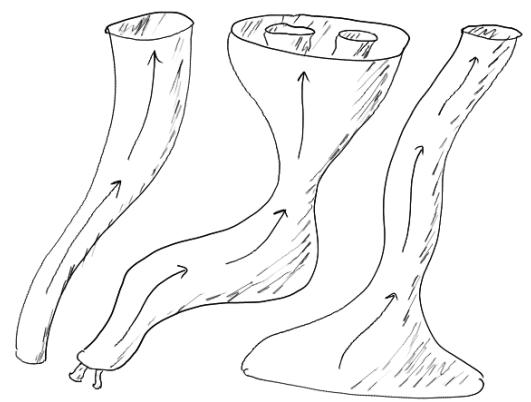

Two-dimensional representation of tubes of a closed outer-oriented 3-form $\boldsymbol{M}$ relativity.

If we were to include a theory of mixtures ${ }^{20}$, we would represent different substances by different matter 3 -forms, which could coexist in the same spacetime region.

The matter 3-form $\boldsymbol{M}$ provides a frame $\boldsymbol{V}$ wherever it doesn't vanish. It is called a Lagrangean or material frame ${ }^{21}$ and defined by

$$
\boldsymbol{V} \cdot \boldsymbol{M}=0, \quad \boldsymbol{V} \cdot \mathrm{d} t=1 .
$$

It represents the correspondence of places at different times defined by simply saying that each part of that body is at rest.

Note that $V$ is not the 4-velocity of matter; the latter is given by the condition $\boldsymbol{V} \cdot \mathrm{d} t_{\mathrm{a}}=1$ in Newtonian relativity and $|\boldsymbol{V} \cdot \boldsymbol{\gamma} \cdot \boldsymbol{V}|=1$ in general relativity, where $\gamma$ is the 4-metric. Equivalently in general relativity it can be defined as

$$
V=\frac{|\gamma|^{-\frac{1}{2}} \vdots M}{M \vdots|\gamma|^{-\frac{1}{2}} \cdot \gamma \cdot|\gamma|^{-\frac{1}{2}} \vdots M}
$$

where $|\gamma|^{-\frac{1}{2}}$ is the inverse volume element associated with the 4-metric, and : represent full contraction (remember that $M$ is a 3 -form).

A matter 3-form cannot be used to define time lapse or distance, owing to its purely differential-geometric nature.

20 e.g. Truesdell 1984 app. 5B. $\quad{ }^{21}$ Smarr et al. 1978; 1980. 
The matter 3-form of a body can also be used to define a volume element on each slice, by decreeing that each unit amount of matter of that body occupies a unit volume.

Once we have a slicing $t$ and a frame $U$, we can project the matter 3-form:

$$
\boldsymbol{J}:=-\hat{\boldsymbol{M}} \equiv-\boldsymbol{U} \cdot \boldsymbol{M}, \quad N:=\overline{\boldsymbol{M}} \equiv \boldsymbol{M}-\mathrm{d} t \wedge(\boldsymbol{U} \cdot \boldsymbol{M}) .
$$

We can speak of the amount of matter contained at time $t$ within a 3-dimensional region on a slice: it's the integral of $N$ (or $\boldsymbol{M}$ ) over that region. We can speak of the rate at which matter is flowing through a stationary outer-oriented 2-dimensional surface on a slice: it's the integral of the outer-oriented 2-form $J$ over that surface. We can also speak of the spacelike velocity of matter with respect to the frame $U$ at time $t$ : it's the vector $v$, tangent to the slice, satisfying

$$
\boldsymbol{v} \cdot \mathrm{d} t=0, \quad \boldsymbol{v} \cdot \boldsymbol{M}=-\boldsymbol{U} \cdot \boldsymbol{M} \text { or } \boldsymbol{v} \cdot N=\boldsymbol{J},
$$

or equivalently, in terms of the Eulerian frame (8),

$$
v:=V-U \text {; }
$$

i.e. it's the difference between the frame $U$ and the Eulerian frame $V$. This is a velocity in the sense of our convention of slicing \& frame, not in the metric sense.

For the latter, we would have to replace the left eq. (8) with a normalization condition.

The outer-oriented 3-form $N$ is a density when it's restricted to a

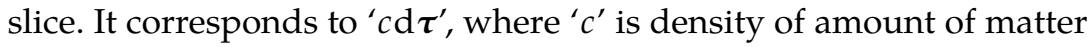
(concentration, amount/volume) and $\mathrm{d} \tau$ a volume element. Thus it could be measured in 'moles' 22 . The outer-oriented 2-form $J$ is related - but doesn't correspond - to ' $c \boldsymbol{v}$ ', where $v$ is the traditional velocity (length/time). This product is the traditional momentum divided by mass, which is identical with flux of amount of matter in continuum mechanics. But, as we'll see, we have to keep these two notions distinct. The 2-form $J$ is matter-flux, but not momentum.

With the projections (10) and the expression for the differential (5), the matter balance (7) can be expressed as

$$
\mathrm{L}_{U} N+\mathrm{d} J=0,
$$

since $\mathrm{d} N \equiv 0$ on a slice by construction. This simple, metric-free formula should be compared with the one involving the covariant derivative 22 ISO 2009a,b; JCGM 2012. 
or several metric volume elements when matter flow is expressed by a 1 -vector or a 1 -form instead ${ }^{23}$.

By specifying a slicing, a frame, and a matter 3-form, we are specifying a differential-geometric kinematics. We're only short of a time lapse and a distance for a kinematics in the traditional sense. The major aim of Newtonian relativity is to specify the causes that lead to a particular kinematic.

We have here pulled the following piece of thread: mathematically defining matter as a three-form, with its conservation (7) or (12). This seems to be most natural and convenient: metric-free and valid in both Newtonian and general relativity. The tangled bundle loosens here.

But alas, it gets more tangled elsewhere. The projection $J$ of the matter 3-form on the frame, eq. (10) left, $_{\text {, }}$ is a 2 -form, and traditionally it would be considered as 'momentum'. As momentum it would be part of the energy-momentum-stress tensor $\boldsymbol{T}$, which has order 2 . This only works if we represent it as a 1-form or a vector. Should we change our representation of the energy-momentum-stress tensor $\boldsymbol{T}$ then, to something of a different order? We'll see in $\S^{* *}$ that there are other reasons to do so indeed. But in Einstein's equations $\boldsymbol{G}=8 \pi \boldsymbol{T}$ this tensor is equated to the Einstein tensor $\boldsymbol{G}$; thus we would need to find a different representation of the latter, too.

\section{Force in Newtonian relativity}

The second piece of thread we're going to pull is force. This is actually a smaller bundle in itself, and we'll examine it from several angles.

*** why no need to speak of conservation

***dynamics comes from stating that the total force and total torque vanish

***affine structure needed, related to Newton's 3rd law. But if we assume only contact forces, it can be expressed locally without parallel transport. Exception: add spacelike surfaces from a 4D point of view

***that would suffice. But 1st law of thermodynamics, needed for thermomechanics, mixes force in energy equation as work.

${ }^{* * *}$ when the constitutive equation for a force 'visibly' depends on space or time variables, as is the case for elastic or inertial forces, the

23 e.g. Rezzolla et al. 2013 eq. (7.205), § 7.3, p. 361; Gourgoulhon 2012 eq. (6.47), § 6.3.2, p. 110. 
amount of work done by the force can be 'read' in a change of that variable. This change is usually called 'energy'. Typical examples are elastic force $-k x$ and elastic energy, the integral of $-k x \dot{x}$ being equal to $-\frac{1}{2} k x^{2}$ no matter how $x$ depends on time; and inertial force $-m \ddot{x}$ and kinetic energy, the integral of $-m \ddot{x} \dot{x}$ being equal to $-\frac{1}{2} m \dot{x}^{2}$ no matter how $x$ depends on time. For viscous forces like $-\eta \dot{x}$ we usually don't speak of 'viscous energy' probably because the integral of $-\eta \dot{x}^{2}$ does not have a closed form. This fact is obviously related to the difference between conservative and non-conservative forces and to the existence of potentials.

\subsection{Constitutive equations}

\section{to be written. See $§ 8.3$}

\subsection{Geometric representation}

In most presentations of the Newtonian mechanics of point masses, force is represented as a vector bound to a point mass. If we take the line integral of this vector along the trajectory of the point mass, we obtain the work made by the force on the mass. In continuum mechanics this picture becomes more complex. We deal with extended bodies, and the total force on a body or on part thereof is obtained by integrating two kinds of forces: bulk forces, also called body forces, which act on a volume element and must be integrated over a three-dimensional region, and surface forces, also called contact forces, which act on an area element of the body's surface and must be integrated over a two-dimensional region.

Let's see what kind of geometric representation these operations suggest.

The fact that a force is to be integrated over a line to obtain work suggests that it's better viewed as a covector-valued object, ready to be integrated over a line without the need of a scalar product ${ }^{24}$.

If a bulk force is to be integrated over a volume, then it should be a covector-valued, outer-oriented 3-form. If we think of it as operating on a matter element (outer-oriented 3-form) instead, then it should be a covector-valued, outer-oriented 3-vector. Here we see the core of one

$\overline{24}$ Burke 1995 § 12; Schouten 1951 § VII.2; van Dantzig 1954 § 2; Burke 1987 chs VI-VII; Bamberg et al. 1990 ch. 7. 
of the questions of $\S 1$ : are we to interpret forces as 'acting' on matter? or as entities that can exist where there's no matter? General relativity suggests the latter interpretation. But we'll see that we can bypass the question of this representation in general relativity, because the notion of bulk force disappears there, leaving only that of surface force.

Surface forces, being integrated over a surface, should be covectorvalued, outer-oriented 2-forms.

The integration of bulk or surface forces, which are covector-valued object, is possible in Newtonian mechanics thanks to the affine structure of its three-dimensional space. This kind of integration is impossible or at best ambiguous with the path-dependent parallel transport of general relativity; this impossibility is related to the absence of a 'conservation law' for 4-momentum ${ }^{25}$.

$* * 1$-form also suggested by principle of virtual work

\subsection{Frame indifference}

\subsection{Work and frames}

\subsection{Inertia as force and affine structure}

*** only affine structure is necessary to transport and sum forces *** static formulation ${ }^{26}$

\subsection{Bulk and surface forces}

\subsection{Four-dimensional generalizations}

*** 4th component, spacelike 3-surface

\subsection{Newtonian relativity}

The main aim of continuum mechanics is to describe how bodies move and deform in spacetime in response to forces.

In the history of science we see two main kinds of scientific theory.

One kind of theory is based on the 'stuff' that is believed to be ultimate and fundamental at the time of conception, although usually proven not to be ultimate some decades later. Examples are vortex theories from the Baroque and contemporary high-energy particle theories.

25 Pauli 1958 § 21; Eddington 1930 § 59; Landau et al. 1994 § 96; also Alderman et al. 1970. 26 cf. van Dantzig 1934 b § 6, p. 652. 
The other kind of theory, humbler and more grandiose at the same time, tries to make allowance for any new phenomena that may be discovered.

Newtonian thermomechanics belongs to the second kind. Its aim is not to describe this or that particular particle or field, or this or that fundamental interaction. Its purpose is the description, within particular ranges and resolutions of space and time, of any kind of material and substance that humans use now or will discover in the future. For this purpose it uses some concepts devised, with their generality, to be able to accommodate as many new phenomena as possible. The main one is the concept of force.

Our exploration cannot only be based on mathematical manipulation of the Einstein equations, but also requires a clear view of the way continuum thermomechanics is used.

Newtonian continuum thermomechanics, which I'm here calling 'Newtonian relativity', has been refined under more than 300 years and has today a crystal-clear, yet far from finished, method and structure:

- Its purpose is the description, within particular ranges and resolutions of space, time, energy, of any kind of material or substance that Man uses now or will discover in the future. This must be contrasted with contemporary particle high-energy physics, whose goal is the description of only those forces and kind of matter believed to be 'ultimate'. The scope of Newtonian continuum thermomechanics is from this point of view wider than that of particle physics.

- For this description it uses five fundamental balance laws and a set of nine physical quantities:

- balances: amount of matter, force, torque, energy, entropy;

- quantities: amount of matter, displacement, temperature, stress, internal energy, heating flux, entropy, body force, body heating.

The quantities and balances above are postulated to apply to any kind of matter or substance. Amount of matter, displacement, temperature are independent quantities. Body force and body heating must be given in any particular problem and express the effect of the surroundings The remaining are dependent quantities.

- Each particular kind of matter, with its different behaviours and responses, is characterized by a set of constitutive equations. These equations express the particular dependence of the dependent quantities on the independent ones, and together with the balances and initial and 
boundary conditions yield a well-posed system of integro-differential equations. Specifically, the constitutive equations give stress, internal energy, heating flux, and entropy as functionals of amount of matter, displacement, temperature. The difference between al kinds of liquids, gases, solid, plasmas resides in the constitutive equations.

Sometimes internal energy is taken and independent, and temperature as dependent with an appropriate constitutive equation.

The separation of balance laws and constitutive equations is fundamental and reflects the aim of continuum mechanics to describe any kind of force and matter that can be discovered, not just those that our century believes to be the only existing ones. We can make a parallel with Hamiltonian mechanics: Hamilton's general equations correspons to the balance laws; particular Hamiltonians correspond to particular constitutive equations.

\section{Newtonian-relativistic continuum mechanics: kinematics}

\section{Newtonian-relativistic continuum mechanics: dynamics}

\subsection{Dynamics with absolute time}

Traditional presentations of Newtonian-relativistic dynamics and of Newton-Cartan theory starts with the notion of absolute time $t_{\mathrm{a}}$, which is a unique slicing of spacetime. We'll presently see that this notion, together with a geometry and a connection on the slices, is mathematically necessary when the notion of force is represented by objects living on 3surfaces. But later we'll consider the possibility of avoiding absolute time. In fact, the notion of simultaneity in Newtonian-relativistic mechanics is inspired by what we simultaneously see, together with the assumption that the velocity of light is practically infinite. But we could also define simultaneity in terms of what we simultaneously hear, for example. How could Newtonian mechanics be formulated in that case? We'll give a possible answer later.

A force on a body at time $t_{\mathrm{a}}$ in Newtonian mechanics has two important characteristics: (1) it is meant to be integrated along the trajectory of every small region of the body, to yield the work done on that region; (2) it is meant to be obtained from the sum of infinitesimal body forces acting over each part of the region, or surface forces over its boundary. 
Owing to the first characteristic, many geometers ${ }^{27}$ have advocated the representation of force as a covector. Their arguments are geometrically beautiful. Further consideration of geometry, however, leads to a still different representation. The reason is that velocity is a frame-dependent object. If we represent forces as covectors, we're then supposing that a frame-independent object should be applied to a frame-dependent one, which feels geometrically unsatisfying. There is a way out. Instead of the velocity of matter we can consider its flow, represented by the matter 3-form $M$. On a 3-surface its current is represented by the flux 2-form $J$. We can let a force operate on this flux, rather than on the velocity. Then a force has to be represented by a 2-vector rather than a 1-covector. The 2 -form flux still depends on a choice of frame, but this point of view can be generalized to four dimensions in a frame-independent way.

The second characteristic says that a body force must be a 2-vectorvalued outer-oriented 3-form, while a surface force a 2-vector-valued outer-oriented 2-form.

The total force on a body is the integral of the body forces over its volume and of the surface forces over its boundary. Since forces are vector-valued objects, this integration is only possible if the slices have an affine structure, that is, a parallel transport with vanishing curvature. From this point of view, the affine structure is demanded by the dynamics, rather than by the kinematics.

Some forces between bodies, like the gravitational force or a purely elastic stress, are moreover defined to depend on a metric, which must therefore be introduced besides the affine structure. It would be interesting to explore whether metric and affine structure could be introduced in terms of these forces instead.

So in the present section let's assume that we have a unique slicing $t \equiv t_{\mathrm{a}}$, representing absolute time, and whose slices have a Euclidean structure: metric $\boldsymbol{g}_{t}$ and flat parallel transport $\nabla_{t}$; the index $t$ will be omitted, but it is important to note that there is no specific relation between metrics and parallel transport on different slices. We are not assuming the existence of a Newton-Cartan connection. A frame $U$ that satisfies $\mathrm{L}_{\boldsymbol{u}} \boldsymbol{g}=0$ is called a rigid frame.

27 Van Dantzig 1954; Burke 1980; 1987; 1995; Bossavit 1991; 1998; Hehl et al. 1999; 2003. 
Indicating the sum of all body forces acting on a body by $f$, and that of the surface forces by $\tau$, the balance of forces states that

$$
\int_{V} f+\oint_{\partial V} \tau=0 \quad \forall V
$$

for each outer-oriented closed 3-volume $V$ within a slice. Note that the result of the integration is a 2 -vector, not a scalar. The local version of the balance is

$$
f+\nabla \tau=0,
$$

where $\nabla$ is the exterior covariant derivative ${ }^{28}$.

The fact that we have to use the exterior covariant derivative, rather than the covariant derivative, may have important consequences later.

\subsection{Interlude: forces and motion - is there a balance of momentum?}

How does the balance of forces relate to motion?

The vast majority of literature and textbook do not speak of balance of forces; they follow a different path that sounds more or less as follows:

There is a law of 'conservation of momentum', which states that the rate of change of momentum of a body region is proportional to the forces exerted on it:

or

$$
" \mathrm{~d}_{t} \int_{V} \rho v=\int_{V} f+\oint_{\partial V} \tau ",
$$

$$
“ \partial_{t}(\rho v)+\operatorname{div}(\rho v \otimes v)=\operatorname{div} \tau+f " .
$$

This equation directly connects forces and motion, and looks like a conservation equation with fluxes and sources. This conservation law is only valid in an inertial frame. We can make it valid in every rigid frame provided we add some 'fictitious forces' looking like

$$
\text { " } \rho\left[-\partial_{t}^{2} x^{\star}+2 \Omega^{\star} \cdot \partial_{t}\left(x-x^{\star}\right)+\left(\partial_{t} \Omega^{\star}-\Omega^{\star 2}\right) \cdot\left(x-x^{\star}\right)\right] ",
$$

in that non-inertial rigid frame.

The presentation above is geometrically unsatisfying, however.

28 Choquet-Bruhat et al. 1996 § Vbis.A.4; Misner et al. 1973 § 14.5; Frankel 2012 § 9.3; see also Segev 2000a,b; Segev et al. 2000; Segev 2002; Segev et al. 2012; Kanso et al. 2007. 
The right-hand side of eq. (15) is frame-indifferent; that is, the definition and measurement of $\tau$ and $f$ do not depend on a choice of frame, inertial or otherwise. For example, the force exerted on a pointlike object attached to a Hookean spring with constant $k$ and elongation $l$ has intensity $k l$ and direction along the elongation. This is a complete description of the force, and the notion of frame is completely irrelevant to it. Similarly for the gravitational force: it is given by $\rho \otimes \gamma$, where $\gamma$ is a frame-indifferent covector field. This field is in turn determined by the amount of matter and mass distribution via $\mathrm{d} \gamma=-4 \pi G \rho$, again a frame-indifferent definition.

The momentum density $\rho v$ on left-hand side of eq. (15) and the 'fictitious forces' (16) require the specification of a frame instead.

Therefore eq. (15), with or without fictitious forces, mixes up frameindifferent and frame-dependent quantities. This doesn't make sense geometrically.

We find a geometrically more satisfying formulation by using some ideas that can possibly be found in a work by Jacob Bernoulli ${ }^{29}$. Consider a small enough body region, so that deformation and torques can be neglected. In a problem of statics in an inertial frame, the balance of forces (13) holds. Now consider a fact and two assumptions:

- a small body region can always be considered at rest in a suitably chosen frame;

- the laws of statics should hold in any frame in which the body region is at rest;

- an inertial frame is a rigid frame at rest on average with respect to the total distribution of matter in the universe, which is mostly associated with the fixed stars.

The consequence of these statements is that the balance of forces (13) is valid in any frame; among these forces we must account for one related to the relative motion of the body region with respect to the total distribution of matter in the universe, approximately identified with the 'fixed stars'.

This force is the inertial force. Let's see how it is expressed in an arbitrary rigid frame $U$; remember that what we call 'time derivative' is the Lie derivative $\mathrm{L}_{U}=: \partial_{t}$. In this frame let the position of the body region be $x(t)$, and let the total distribution of matter in the universe have centre of mass at $x^{\star}(t)$ and average instantaneous rotation given by

29 Bernoulli 1703; see Truesdell 1968 p. 104; Noll 1963; Truesdell 1991 § I.13. 
the 2-form $\Omega^{\star}(t)$; in $\S$ A I explain how they are determined by the mass distribution through a sort of Mach's principle. Then the inertial force is the covector-valued 3-form

$$
f^{\star}:=-\rho \otimes\left(\Omega^{\star} \cdot g^{-1} \cdot \Omega^{\star}-\partial_{t} \Omega^{\star}-2 \Omega^{\star} \partial_{t}+g \partial_{t}{ }^{2}\right) \cdot\left(x-x^{\star}\right) .
$$

The definition of this force requires a metric on each slice. The definition of its source, see $\S \mathrm{A}$, also requires a parallel transport on each slice. But it does not require a 4-dimensional connection on the whole spacetime. If we choose a rigid frame in which the total matter of the universe does not rotate on average, $\Omega^{\star}=0$, and moves with uniform velocity, $\partial_{t} x^{\star}=$ const, then we recover the familiar expression $f^{\star}=-\rho \boldsymbol{g} \cdot \partial_{t}{ }^{2} x$ - the $\boldsymbol{g}$ is necessary to transform a vector into a covector. This kind of rigid frame is called inertial. The use of a rigid frame is not necessary; a frame-independent expression of the inertial force is given below.

Including the inertial force in the sum of all other body forces $f$, the balance of forces (13) includes the familiar Newtonian equations of motion. It is the inertial force that in most physical situations links kinematics and dynamics, specifically by linking forces upon a body to its motion with respect to the rest of matter in the universe. But there are also situations in which inertia is inessential. For example, consider a small body of negligible mass attached to a Hookean spring inside a fluid, the other end of the spring being at rest with respect to the fluid. The forces acting on the body are the Hookean and the viscous ones. In a suitable frame of reference the balance of forces is $-k x-\eta \partial_{t} x=0$, leading to a specific motion $x(t)=\mathrm{e}^{-k t / \eta} x(o)$ with respect to the fluid. In this example the inertial force plays no role and it is not important whether the frame is inertial or not.

As regards 'conservation of momentum', we see that the timederivative of momentum in Newtonian mechanics is not a quantity with a general geometric meaning: it is the simplified form assumed by a particular force in a particular frame. From this point of view conservation of momentum is not a general geometric notion in Newtonian-relativistic mechanics.

The inertial force on the mass $M$ can be written in a frame-free way as $-\mathbf{g}^{-1} \cdot \mathrm{L}_{\boldsymbol{U}^{\star}}\left(\boldsymbol{U}^{\star} \cdot \boldsymbol{M}\right)$, where $\boldsymbol{U}^{\star}$ is a vector field transverse to the slices, defined in $\S$ A. Some forces, like inertial, viscous, and electromagnetic ones, require objects transverse to the slicing for their definition, whereas others, like elastic or gravitational ones, don't. This suggests that the 
presentation in terms of an absolute-time slicing can be geometrically improved upon.

\subsection{Balance of energy}

To each matter element on each slice is also associated an internal energy density $\epsilon$, represented by an outer-oriented 3-form proportional to the mass 3-form $N$. The balance of energy states that the change in the total energy in a 3-volume $V$ on a slice must come from the energy transported thither or thence, from the work done by the forces, and from body and surface heating, represented by outer-oriented 3-form and 2-form:

$$
\partial_{t} \epsilon+\mathrm{d}(v \cdot \epsilon)=v \cdot f+\mathrm{d}(\boldsymbol{v} \cdot \tau)+h+\mathrm{d} \boldsymbol{q},
$$

with $\partial_{t} \epsilon \equiv \mathrm{L}_{U} \epsilon$. The rate of change of kinetic energy is simply the work done by the inertial force, and is therefore included in the term $v \cdot f$. The traditional expression with ' $\partial_{t}+v \cdot \nabla$ ' appears if we write the 3 -form $\epsilon N$ as a multiple of the volume element $\sqrt{g}$ - one more example of a unnecessary use of metric.

If we assume that body forces and body heating vanish, then we can rewrite this equation as

$$
\partial_{t} \epsilon+\mathrm{d}(v \cdot \epsilon-v \cdot \tau-q)=0,
$$

which suggests that energy can also be considered like an outer-oriented closed 3-form, with a flux 2-form

$$
q \equiv-v \cdot \epsilon+v \cdot \tau+q .
$$

\subsection{Dynamics without absolute time}

The formulation of dynamics of $\S 7.1$ has the advantage of being frameindifferent: inertial frames don't have a special status, and inertia is just one force among many others. But that formulation still has several interrelated unpleasant features:

- a special absolute-time slicing is used,

- forces, energy, energy fluxes are only defined on the slices of that slicing,

- forces are meant to act on velocities, which are frame-dependent,

- a flat parallel transport is needed to integrate the forces. 
A first step in lifting forces from their plain existence on slices to a fourfilling existence in spacetime, and in unchaining them from frame-dependent velocities, is to assume that they act directly on the fourdimensional mass flux expressed by the matter 3-form. This requires that they have outer-oriented 3-vector values. It also means that their physical dimension acquires a $\left[\mathrm{mass}^{-1}\right]$ factor.

A 3-vector has four components. The additional component of a force is interpreted, both in Newtonian ${ }^{30}$ and Lorentzian ${ }^{31}$ relativity, as the combination of power and heating. Thus we arrive at the notion of heating-force tensor, which corresponds to the 'four-force' of Lorentzian relativity.

In this new point of view a surface force - 4-stress - is a outer-oriented3-vector-valued outer-oriented 3-form. Contracted with a matter 3-form it yields another outer-oriented 3-form, which could be interpreted as the spacetime flux of some quantity. From general relativity we foresee that this quantity is the energy flux.

낭 To be added: formulation with 2-point energy-momentum-stress tensor ${ }^{32}$

\subsection{Decoupling force from body}

In Newtonian relativity, force is something experienced by a body, rather than something in spacetime. But there are signs that forces need to be decoupled also in Newtonian relativity.

\subsection{An interesting development?}

20 The idea in this section is probably seriously wrong

This leads to some interesting developments. Interpret a 4-stress $\boldsymbol{T}$ as an object which contracted with a matter 3-form $\boldsymbol{M}$ yields its corresponding energy-flux outer-oriented 3-form $\boldsymbol{E}$ :

$$
\boldsymbol{E}=\boldsymbol{T} \cdot \boldsymbol{M}
$$

This is similar to the ${ }^{*} \boldsymbol{T}^{\prime}$ of Misner et al. ${ }^{33}$, with the difference that $\boldsymbol{T}$ is 3-covector valued.

\footnotetext{
30 Truesdell et al. 1960 § 152-154, 288-289; Grot et al. 1966a § 2.3. ${ }^{\mathbf{3 1}}$ Eckart 1940b; Grot et al. 1966a § 2.3; Maugin 1978a. 32 Truesdell et al. 1960 § 288. $\quad 33$ Misner et al. 1973 ch. 15 .
} 
Now postulate that energy flux must always be conserved,

$$
\mathrm{d} \boldsymbol{E}=0 .
$$

We obtain a set of two balances and a relation between them:

$$
\mathrm{d} M=0, \quad \mathrm{~d} \boldsymbol{E}=0, \quad \boldsymbol{E}=\boldsymbol{T} \cdot \boldsymbol{M} .
$$

Question: does this system imply Einstein's equations? The point is that we can now introduce a four-dimensional exterior covariant derivative $\nabla$ and combine the equations above as follows:

$$
\mathrm{d} \boldsymbol{E}=\mathrm{d}(\boldsymbol{T} \cdot \boldsymbol{M})=\boldsymbol{\nabla} \boldsymbol{T} \cdot \boldsymbol{M}-\boldsymbol{T} \cdot \mathrm{d} \boldsymbol{M}=\boldsymbol{\nabla} \boldsymbol{T} \cdot \boldsymbol{M}=0
$$

and asking this to hold for every $\boldsymbol{M}$, we have

$$
\nabla T=0,
$$

which corresponds to Misner et al.'s ' $\boldsymbol{d}^{*} \boldsymbol{T}=0^{\prime 34}$.

This point of view seems to say that any exterior covariant derivative $\nabla$ will do - possibly with additional restrictions coming from restrictions on positivity of mass and energy fluxes. Such a possibility is not so strange when we consider that Einstein's equations can be rewritten, in a completely equivalent way, in terms of a flat connection with non-vanishing torsion, or a mixture of the two ${ }^{35}$. It seems that what's fundamental here is the use of a derivation operator with the properties of an exterior covariant derivative, rather than the use of a covariant derivative or parallel transport per se.

Finally, Einstein's equations $\boldsymbol{G}=\boldsymbol{T}$ could simply be interpreted as the most general integral of the equation $\boldsymbol{\nabla T}=0$. This possibility is related to an interesting remark by Truesdell:

When the field theories were discovered in the eighteenth century, solutions in arbitrary functions such as those presented in this subchapter were sought earnestly, but, for the most part, sought in vain. In the nineteenth century, researches on partial differential equations turned away from such general solutions so as to concentrate upon boundary-value problems. When, in the twentieth century, the general solutions were at last obtained, scarce attention was paid to them, and

34 Misner et al. 1973 ch. 15.35 De Andrade et al. 2000; Arcos et al. 2004; Aldrovandi et al. 2013; Pereira 2014; Cai et al. 2016. 
to this day they remain virtually unknown. Though so far they have been used but rarely, they might turn out to be illuminating in studies of underdetermined systems, where the conventional viewpoint of partial differential equations has gained little. ${ }^{36}$

\section{난 To be added: refs to Finzi, Beltrami}

ㄴ. To be added: discussion about pp. 18-19 of ${ }^{37}$ : use differential forms for Gauss-Green theorem, instead of covariant derivative

\section{General relativity}

\subsection{Aims}

General relativity gives us the equation $\boldsymbol{G}=\kappa \boldsymbol{T}$; for some time after its proposal it was not clear which were independent, dependent, and boundary-condition quantities; a distinction that is necessary to set up a well-posed boundary-value problem. Misner et al. ${ }^{38}$ discuss this question at length.

The Einstein equations are clearly suited for a continuum description of matter, not a particulate one: because they involve the notion of stress, which is an exquisitely continuum notion. And since stress is the staple of constitutive equations in Newtonian mechanics, and does not commit to specific forms of matter or forces, then the Einstein equations do not commit to specific forms of matter or forces either, unlike particle-physics theories. From this point of view the Einstein equations appear as a harmonization of a part of the Newtonian balance laws and of geometry. They - especially the stress appearing in them - still need constitutive equations.

The Einstein equations don't include the balances of amount of matter (see $\S 4$ ) and entropy ${ }^{39}$. This fact is very clear in numerical relativity ${ }^{40}$.

\section{$8.23+1$ formulation}

Spacetime can be sliced and a frame introduced just like in the Newtonian case of $\S 3$.

36 Truesdell et al. 1960 p. $594 . \quad{ }^{37}$ Weatherall 2017. $\quad{ }^{38}$ Misner et al. 1973 ch. $21 . \quad{ }^{39}$ Eckart 1940b; Misner et al. 1973 ch. 22. 40 Disconzi 2014 p. 1918; Wilson et al. 2007 § 2.2; Gourgoulhon 2012 § 6.3.2; Baumgarte et al. 2010 ch. 5; Rezzolla et al. 2013 § 2.3. 
The Einstein equations can be decomposed by projection along the slices $t$ and the frame $V$, giving rise to a part parallel to the surfaces with $3 \times 3$ real components, a part parallel to the vector field with 1 component, and a mixed part with 3 components. Our freedom in choosing the slicing $t$ and the frame $V$ appears in the decomposed equations as the presence of and undetermined 'lapse' function and an undetermined 'shift' vector field. When a metric is present we can choose the frame $V$ to be the unit normal to the 3-surfaces. This choice corresponds to a constant unit lapse function and a vanishing shift vector ${ }^{41}$. Other choices of lapse and shift are more convenient for the initial-value problem, for example because they can correspond to a slicing that covers most of spacetime while avoiding singularities ${ }^{42}$. But our present discussion is mainly conceptual; we therefore use the simplest lapse and shift.

The decomposed Einstein equations can be found compactly written in a number of texts ${ }^{43}$. The decomposition of the Einstein tensor $\boldsymbol{G}$ yields the 3-metric $\boldsymbol{g}=\left(g_{a b}\right)$ of the 3-surfaces, having inverse $\boldsymbol{g}^{-1}=\left(g^{a b}\right)$, with its compatible connection $\nabla$ having Ricci curvature $\boldsymbol{R}=\left(R_{a}^{b}\right)$, and the extrinsic curvature $\boldsymbol{K}=\left(K_{a}{ }^{b}\right)$ of the 3-surfaces. The decomposition of the energy-momentum-stress tensor $\boldsymbol{T}$ yields the internal energy $\epsilon$, energy flux $\boldsymbol{q}$, and spatial stress $\boldsymbol{\tau}=\left(\tau_{a}^{b}\right)$ :

$$
\boldsymbol{T}=\epsilon \mathrm{d} t \otimes \boldsymbol{V}+\boldsymbol{q} \otimes \boldsymbol{V}+\mathrm{d} t \otimes\left(\boldsymbol{q} \cdot \mathbf{g}^{-1}\right)+\boldsymbol{\tau} .
$$

The Einstein equations $\boldsymbol{G}=8 \pi \boldsymbol{T}$ are decomposed into the evolution equations 나 some $\pi$ factors may be missing!

$$
\begin{aligned}
& \partial_{t} \boldsymbol{g}=-2 \boldsymbol{K} \cdot \boldsymbol{g}, \\
& \partial_{t} \boldsymbol{K}=\boldsymbol{K} \operatorname{tr} \boldsymbol{K}-2 \boldsymbol{K} \cdot \boldsymbol{K}+\boldsymbol{R}+4 \pi(\operatorname{tr} \boldsymbol{\tau}-\epsilon)-8 \pi \boldsymbol{\tau}
\end{aligned}
$$

or

$$
\begin{aligned}
& \partial_{t} \boldsymbol{g}=-2 \boldsymbol{K} \cdot \boldsymbol{g} \\
& \partial_{t} \boldsymbol{K}=\boldsymbol{K} \operatorname{tr} \boldsymbol{K}-\frac{1}{4}(\operatorname{tr} \boldsymbol{K})^{2}-2 \boldsymbol{K} \cdot \boldsymbol{K}+\frac{1}{4} \boldsymbol{K}: \boldsymbol{K}+\boldsymbol{R}-\frac{1}{4} \operatorname{tr} \boldsymbol{R}-\boldsymbol{\tau}+\frac{1}{2} \operatorname{tr} \boldsymbol{\tau}
\end{aligned}
$$

and the constraint equations

$$
\begin{aligned}
16 \pi \epsilon & =(\operatorname{tr} \boldsymbol{K})^{2}-\boldsymbol{K}: \boldsymbol{K}+\operatorname{tr} \boldsymbol{R}, \\
8 \pi \boldsymbol{q} & =\nabla \cdot\left(\boldsymbol{K}^{\top}-\mathbf{I} \operatorname{tr} \boldsymbol{K}\right) .
\end{aligned}
$$

41 Smarr et al. 1978; 1980. $\quad 42$ Smarr et al. 1978. $\quad 43$ Wilson et al. 2007 § 1.3; Gourgoulhon 2012 § 4.3.2; Alcubierre 2008 ch. 2; Rezzolla et al. 2013 § 7.2.2. 
Note that

$$
\boldsymbol{K} \cdot \boldsymbol{g}=-\frac{1}{2} \partial_{t} \boldsymbol{g} \equiv-\frac{1}{2} \mathrm{~L}_{V} \boldsymbol{g} \equiv-\frac{1}{\sqrt{\mathbf{g}}} \mathrm{L}_{V} \sqrt{\mathbf{g}} \equiv-\nabla \cdot * * *
$$

It's interesting that the '3-momentum' in relativity appears as the divergence of a 3-tensor - just like the force from a stress.

To these equations we must add the balance of amount of matter (12):

$$
\partial_{t} N=-\mathrm{d} \boldsymbol{J} \quad \text { or } \quad \partial_{t} N-\operatorname{tr} \boldsymbol{K} N=-\nabla \cdot \boldsymbol{J},
$$

where in the second equation $N$ and $J$ are interpreted as a scalar and a vector. In these expressions $\partial_{t} \equiv \mathrm{L}_{V}$. (Conservation of matter is not enforced by the Einstein equations ${ }^{44}$. Curiously there was at first some misunderstanding about the independence of this equation: see for example von Laue's ${ }^{45}$ statement.)

This gives us a total of $6+6+1+3+1$ real-component equations for a total of $6+6+6+1+3+1+3+1$ real components of the fields $\mathbf{g}, \boldsymbol{K}, \boldsymbol{\tau}$, $\epsilon, \boldsymbol{q}, N, \boldsymbol{J}$, and temperature $\theta$. Then we need $6+1+3$ equations, which turn out to be, as in the Newtonian case, the constitutive functional equations (33) for the energy-momentum-stress:

$$
\begin{aligned}
& \epsilon=\epsilon[N(\cdot), \boldsymbol{J}(\cdot), \theta(\cdot), \mathbf{g}(\cdot), \boldsymbol{K}(\cdot)], \\
& \boldsymbol{q}=\boldsymbol{q}[N(\cdot), \boldsymbol{J}(\cdot), \theta(\cdot), \boldsymbol{g}(\cdot), \boldsymbol{K}(\cdot)], \\
& \boldsymbol{\tau}=\boldsymbol{\tau}[N(\cdot), \boldsymbol{J}(\cdot), \theta(\cdot), \boldsymbol{g}(\cdot), \boldsymbol{K}(\cdot)]
\end{aligned}
$$

(the dependence on $\boldsymbol{K}(\cdot)$ is redundant, since it's a functional of $\boldsymbol{g}(\cdot)$, and these equations are already functionals of $\boldsymbol{g}(\cdot)$; but I leave the explicit dependence for clarity).

(The 4-velocity is determined by the matter flux $J$ and the 4-metric by eq. (9).)

\subsection{Constitutive equations}

This section is a little disconnected from the rest. Must be better integrated

The balances of amount of matter, force, energy give a system of $1+3+1$ real-valued equations. They involve at least $4+3+6+5$ real-valued

44 e.g. Eckart 1940b; Gourgoulhon 2012 § 6.3.2; Baumgarte et al. 2010 § 5.2; Rezzolla et al. 2013 § 7.3; Misner et al. $1973 \S 22.2 . \quad 45$ Von Laue 1949 p. 503. 
components: matter density and flux, body force and stress, energy density and flux, and body supply of energy. The independent variables in these equations are usually assumed to be the four components of the matter 3-form - that is, density of amount of matter and flux of amount of matter (velocity) - and the temperature. Sometimes the energy density is exchanged with the temperature in the role of independent variable.

The balance laws alone are therefore not sufficient to determine the evolution of all fields. Moreover, although the balances are assumed to hold for all forms of matter, different kinds of matter behave in different ways.

Constitutive equations are equations that give the dependent fields as functions or functionals of the independent ones. They express the different behaviours of different kinds of matter, and together with the balance laws yield a closed system of differential or integro-differential equations.

The most important constitutive equation in our discussion is that for the 4-stress. All 4-components of the stress $\boldsymbol{\tau}$ are in the simplest cases functionals of the matter 3-form $\boldsymbol{M}$, the internal energy $\epsilon$ or temperature $\theta$, and the 3-metric $\mathbf{g}$ :

$$
\boldsymbol{T}=\boldsymbol{T}[\boldsymbol{M}(\cdot), \theta(\cdot), \boldsymbol{g}(\cdot)],
$$

or, in a given slicing and frame,

$$
\boldsymbol{T}=\left(\begin{array}{ll}
\epsilon[N(\cdot), \boldsymbol{J}(\cdot), \theta(\cdot), \boldsymbol{g}(\cdot)] & \boldsymbol{q}[N(\cdot), \boldsymbol{J}(\cdot), \theta(\cdot), \boldsymbol{g}(\cdot)] \\
\boldsymbol{q}[N(\cdot), \boldsymbol{J}(\cdot), \theta(\cdot), \boldsymbol{g}(\cdot)] & \boldsymbol{\tau}[N(\cdot), \boldsymbol{J}(\cdot), \theta(\cdot), \boldsymbol{g}(\cdot)]
\end{array}\right)
$$

(these functionals indirectly include a possible dependence on the extrinsic curvature $\boldsymbol{K}$, because it's the 'time derivative' of the 3-metric; see previous section).

The dependence on the metric can be complicated: for example, the stress at a point can depend on the difference between the 3-metric there and the metric from previous 3-surfaces, transported by the velocity $u$ associated with the matter flux ${ }^{46}$. The difference can be non-vanishing even if the 3-metric is Euclidean on all 3-surfaces. The only difference between Newtonian and general relativistic mechanics is that all 3-metrics are flat in the former but not in the latter.

There are many studies of specific constitutive equations in general relativity, from perfect fluids to materials with elasticity dependent on electromagnetism, temperature, and heat conduction. There are

$\overline{46}$ Grot et al. 1966a; Carter et al. 1972. 
also studies on general symmetry principles for the formulation of constitutive equations. Unfortunately many of these works don't know of one another's existence. See especially the extensive studies and results by Maugin et al. ${ }^{47}$, Carter et al. ${ }^{48}$, the early and very insightful study by Eckart $^{49}$, and others articles and books ${ }^{50}$.

\subsection{Parentetical remark: divergence of energy-momentum-stress}

A strange point appears in most general-relativity texts I've seen, especially for numerical relativity. They remark that the balance equations $\bar{\nabla} \cdot \boldsymbol{T}=0$ are a consequence of the Einstein equations $\boldsymbol{G}=8 \pi \boldsymbol{T}$, and therefore solving the latter automatically yields the former. But to find the numerical solutions they actually use the former!

The balance $\bar{\nabla} \cdot \boldsymbol{T}=0$ is indeed contained in eqs (27)-(29). For example, combining the time derivative of the constraint equation (29b) and the 3-divergence of the evolution equation (27b) we obtain the spatial part of $\bar{\nabla} \cdot \boldsymbol{T}=0$ : the balance of momentum. A quick check of such combination of (29b) and (27b) shows in fact the appearance of the time-derivative of $q$ and the divergence of the 3-stress. A similar combination yields the balance of energy:

$$
\begin{gathered}
\partial_{t} \epsilon-\epsilon \operatorname{tr} \boldsymbol{K}+\nabla \cdot \boldsymbol{q}-\boldsymbol{K}: \boldsymbol{\tau}=0 \\
\partial_{t} \boldsymbol{q}-\boldsymbol{q} \operatorname{tr} \boldsymbol{K}+\nabla \cdot \boldsymbol{\tau}^{\top}=0 .
\end{gathered}
$$

In these equations the convection terms, usually kept separate in the Newtonian case, are included in $\boldsymbol{q}$ and $\boldsymbol{\tau} \boldsymbol{\top}$. The terms proportional to tr $\boldsymbol{K}$ are absent in the Newtonian case.

The reason for using the divergence equation $\bar{\nabla} \cdot \boldsymbol{T}=0$ is clearly explained by Frittelli ${ }^{51}$ (the only work I've found that explains this). The constraint equations (29) should be enforced on every 3-surface, i.e. for all times $t$, while the metric and extrinsic curvature are evolved by eqs (27). If we did so there would be no need to use $\bar{\nabla} \cdot \boldsymbol{T}=0$ in the solution.

But it would be more convenient if the constraints (29) could be enforced on one 3-surface only, for example the initial one, and be

\footnotetext{
47 Maugin 1971a,b; Maugin et al. 1972a,b; Maugin 1973; 1974a,b; 1978b,a,c,d,e. 48 Carter et al. 1972; Carter 1973; Carter et al. 2006. ${ }^{49}$ Eckart 1940b. $\quad{ }^{\mathbf{5 0}}$ Bressan 1964; Lianis 1973; Bertotti et al. 1984; Hiscock et al. 1985; Anile et al. 1989; Geroch et al. 1991; Herrmann et al. 2000; Bossavit 2001; Geroch 2001; Gourgoulhon 2012; Alcubierre 2008; Choquet-Bruhat 2009; Baumgarte et al. 2010; Rezzolla et al. 2013; Disconzi 2014; Pimentel et al. 2016; Disconzi et al. 2017. $\quad{ }^{\mathbf{5 1}}$ Frittelli 1997.
} 
automatically satisfied on all other 3-surfaces, i.e. at all subsequent times. It turns out that the divergence equation $\bar{\nabla} \cdot \boldsymbol{T}=0$ does exactly this: it makes the constraints automatically satisfied at all times if they are satisfied at the initial time.

Loosely speaking, the system

$$
\left\{\begin{array}{l}
\text { evolution equations (27) } \\
\text { constraint equations (29) for all } t
\end{array}\right.
$$

is equivalent to the system

$$
\left\{\begin{array}{l}
\text { evolution equations (27) } \\
\text { divergence equation } \bar{\nabla} \cdot \boldsymbol{T}=0 \\
\text { constraint equations (29) for initial } t \text { only }
\end{array}\right.
$$

and the latter is more convenient to solve.

\subsection{How to solve the decomposed equations}

Let us consider how equations (27)-(32) could be solved.

Imagining a rough numerical timestepping scheme, in which for example eq. (27a) becomes

$$
\mathbf{g}(t+\Delta t) \approx \mathbf{g}(t)-2 \boldsymbol{K}(t) \cdot \mathbf{g}(t) \Delta t,
$$

it seems that the equations (27)-(32) can be solved this way:

1. Choose all fields such as to satisfy the constraints (29) and constitutive equations (32) on an initial 3-surface at time $t$.

2. Calculate metric $\mathbf{g}$, extrinsic curvature $\boldsymbol{K}$, density of amount of matter $N$ at time $t+\Delta t$ from the evolution eqs (27) and balance (31):

$$
\begin{aligned}
\partial_{t} \boldsymbol{g} & =-2 \boldsymbol{K} \cdot \boldsymbol{g}, \\
\partial_{t} \boldsymbol{K} & =\boldsymbol{K} \operatorname{tr} \boldsymbol{K}+\boldsymbol{R} \cdot \boldsymbol{g}^{-1}+4 \pi(\operatorname{tr} \boldsymbol{\tau}-\epsilon)-8 \pi \boldsymbol{\tau}, \\
\partial_{t} N & =-\mathrm{d} \boldsymbol{J} .
\end{aligned}
$$

Note that these evolution equations require, in particular, the flux of amount of matter $J$, energy $\epsilon$, stress $\tau$ at time $t$. 
3. Calculate the flux of amount of matter $J$, temperature $\theta$, energy $\epsilon$, energy flux $q$, stress $\tau$ at time $t+\Delta t$ from the system of constraints (29) and constitutive eqs (32), given the newly found $\boldsymbol{g}, \boldsymbol{K}$, $N$ :

$$
\begin{aligned}
16 \pi \epsilon=(\operatorname{tr} \boldsymbol{K})^{2} & -\boldsymbol{K}: \boldsymbol{K}+\boldsymbol{R}: \mathbf{g}^{-1}, \quad 8 \pi \boldsymbol{q}=\nabla \cdot\left(\boldsymbol{K}^{\top}-\mathbf{I} \operatorname{tr} \boldsymbol{K}\right), \\
\epsilon & =\epsilon[N(\cdot), \boldsymbol{J}(\cdot), \theta(\cdot), \boldsymbol{g}(\cdot), \boldsymbol{K}(\cdot)], \\
\boldsymbol{q} & =\boldsymbol{q}[N(\cdot), \boldsymbol{J}(\cdot), \theta(\cdot), \boldsymbol{g}(\cdot), \boldsymbol{K}(\cdot)], \\
\boldsymbol{\tau} & =\boldsymbol{\tau}[N(\cdot), \boldsymbol{J}(\cdot), \theta(\cdot), \boldsymbol{g}(\cdot), \boldsymbol{K}(\cdot)] .
\end{aligned}
$$

Note that this system implicitly yields, in particular, the flux of amount of matter $J$, energy $\epsilon$, stress $\tau$ needed in the next timestep; see step above.

Now we have all fields at time $t+\Delta t$.

4. Set $t+\Delta t \rightarrow t$, go to step 2..

It's important to remark that the motion of matter, expressed by the matter flux $J$ in this particular reference frame and slicing, is determined in an implicit way in the scheme above. It would be worth investigating how geodesic equations ${ }^{52}$ peep out from the present scheme.

This scheme can be generalized to more complex temporal dependences in the constitutive equations, like dependence on derivatives, memory, etc..

\subsection{A possible reduction and reinterpretation: 'hot metric'}

An interesting aspect of the equations above is that energy density and energy flux seem to have an intermediate role. We could think of solving the constraint and constitutive equations of step 3. for matter density $N$ and matter flux $\boldsymbol{J}$ in terms of the 4-metric $(\boldsymbol{g}, \boldsymbol{K})$ and the temperature $\theta$; this dependence could involve integro-differential operators. The balance of amount of matter $\partial_{t} N=-\mathrm{d} J$ would then become an evolution equation for the temperature. The constitutive equation for the stress would also be reduced.

$\overline{52 \text { Geroch et al. 1975; Weatherall } 2011 .}$ 
We would be left with the system

$$
\begin{gathered}
\boldsymbol{K}:=-\frac{1}{2} \partial_{t} \boldsymbol{g} \cdot \boldsymbol{g}^{-1}, \\
\partial_{t} \boldsymbol{K}=\boldsymbol{K} \operatorname{tr} \boldsymbol{K}-\frac{1}{4}(\operatorname{tr} \boldsymbol{K})^{2}+\frac{1}{4} \boldsymbol{K}: \boldsymbol{K}+\frac{3}{4} \boldsymbol{R} \cdot \boldsymbol{g}^{-1}+4 \pi \operatorname{tr} \boldsymbol{\tau}-8 \pi \boldsymbol{\tau}, \\
\partial_{t} N[\boldsymbol{g}(\cdot), \boldsymbol{K}(\cdot), \theta(\cdot)]=-\mathrm{d} J[\boldsymbol{g}(\cdot), \boldsymbol{K}(\cdot), \theta(\cdot)], \\
\boldsymbol{\tau}=\boldsymbol{\tau}[\boldsymbol{g}(\cdot), \boldsymbol{K}(\cdot), \theta(\cdot)],
\end{gathered}
$$

a set of integro-differential equations for the 3-metric $g$, extrinsic curvature $\boldsymbol{K}$, and the temperature $\theta$; or integro-differential for the 3-metric $\boldsymbol{g}$ and the temperature $\theta$. This system seems to say that

- density of amount of matter $N$, flux of amount of matter $J$, energy density $\epsilon$, and energy flux $q$ are temperature-dependent manifestations of the 4-metric;

- the 4-metric can interact with itself in different ways, expressed by particular temperature-dependent constitutive functional equations for $N, \boldsymbol{J}, \boldsymbol{\tau}$.

Would this satisfy Einstein? How to interpret the 'temperature of the metric'?

\section{Appendices}

\section{A Addendum: inertial force from mass}

\section{Refer to Weatherall53.}

Inertial force is presented here as the force exerted from the total matter in the universe. It is proportional to the change, per unit time, in the momentum seen from a frame representing a sort of average position of the total matter: the frame of a rigid body with the same mass centre and second moment, expressed by the Euler tensor.

Suppose that the 3-form $N^{\star}$ representing the total matter density in the universe is well-defined. Now consider the slice $E_{t}$ at time $t$ with its affine structure. The mass centre of the matter distribution at each time $t$ is

$$
P^{\star}(t):=\int_{E_{t}} P \frac{N^{\star}(P)}{\int_{E_{t}} N^{\star}} ;
$$

this integration is a convex combination and makes sense with the affine structure.

53 Weatherall 2017. 
A vector field can be defined at each place $P$ by $P \mapsto P-P^{\star}(t)$ thanks to the affine structure. The Euler tensor ${ }^{54}$ of the mass distribution with respect to its mass centre is

$$
\boldsymbol{e}(t):=\int_{E_{t}}\left(P-P^{\star}(t)\right) \otimes\left(P-P^{\star}(t)\right) \otimes N^{\star}(P) .
$$

This tensor-valued integration is possible thanks to the affine structure of the space slice.

The Euler tensor is symmetric and, if the total mass distribution is enough well-behaved, it is also positive. It has three principal axes along vectors $\boldsymbol{a}_{i}(t)$ with respect to the metric $\boldsymbol{g}$, defined by the generalized eigenvalue equation

$$
\left[\boldsymbol{e}(t)-\lambda_{i}(t) \mathbf{g}\right] \cdot \boldsymbol{a}_{i}(t)=0 .
$$

We define the 'frame of the fixed stars' $U^{\star}$ as that frame that maps the principal axes on all slices into themselves, and in which the mass centre is stationary. These conditions are expressed by

$$
\mathrm{L}_{\boldsymbol{U}^{\star}}\left[\boldsymbol{e}(t)-\lambda_{i}(t) \boldsymbol{g}\right]=0,\left.\quad \boldsymbol{U}^{\star}\right|_{P^{\star}(t)}=\dot{P^{\star}} .
$$

The inertial force field upon a mass density $\boldsymbol{M}$ is then defined as the opposite of the change in momentum as seen from the fixed-star frame:

$$
-\mathbf{g}^{-1} \cdot \mathrm{L}_{U^{\star}}\left(\boldsymbol{M} \cdot \boldsymbol{U}^{\star}\right) \equiv-\mathbf{g}^{-1} \cdot\left(\mathrm{L}_{U^{\star}} \boldsymbol{M}\right) \cdot U^{\star} .
$$

\section{B Bits and pieces}

We have seen that a velocity $\boldsymbol{v}$ can be assigned to a mass 3-form $\boldsymbol{M}$ by eq. (8), if a slicing $t$ and a frame $U$ are provided. Can we relate power to force and to the mass-flux 2 -form $J$ directly? thus avoiding the use of a slicing? In this case force has to be contracted with an outer-oriented 3-covector, so it must be an object with outer-oriented 3-vector values. If a volume element is given, there is a unique correspondence between covectors and outer-oriented 3-vectors. In mechanics there's always the metric volume element $\sqrt{g}$ sneaking around, so it's quite possible that the covector-valued conception of force is tacitly using a metric volume element.

$\mathbf{5 4}$ Truesdell 1991 § I.10. 
** must be 3-vector-valued to act directly on mass 3-form rather than a vector-velocity derived from it. Stress must be integrated over 3-regions.

**Once the 4-stress acts on the mass 3-form, it yields an energy 3-form.

**If we say that the total energy flux must vanish, independently on the mass 3-form? then we can say that the total stress must vanish? and interpret Einstein's equations as $\boldsymbol{G}-\boldsymbol{T}=0$ ? Then $\boldsymbol{G}$ would be the stress associated with the gravito-inertial force? Compare ${ }^{55}$, with the difference that we need to consider $\boldsymbol{T}$ (their ${ }^{* *} \boldsymbol{T}^{\prime}$ ) as 3-vector-valued, rather than simply 1-vector-valued. Then

$$
\begin{aligned}
0 & =\mathrm{d}(\boldsymbol{M} \cdot \boldsymbol{T}) \\
& =(\mathrm{d} \boldsymbol{M}) \cdot \boldsymbol{T}+\boldsymbol{M} \cdot \boldsymbol{\nabla} \boldsymbol{T} \\
& =0+\boldsymbol{M} \cdot \boldsymbol{\nabla} \boldsymbol{T}
\end{aligned}
$$

and asking this to hold for every $\boldsymbol{M}$, we have $\boldsymbol{\nabla} \boldsymbol{T}=0$ (their ' $\boldsymbol{d}^{*} \boldsymbol{T}=0^{\prime}$ ).

In Newtonian mechanics we can choose frames that preserve the Euclidean metric of the slices: $\mathrm{L}_{\boldsymbol{U}} \boldsymbol{g}=0$, called rigid frames. In general relativity it is generally impossible to choose a vector field this way, but we can choose one normal to the slices with respect to a spacetime 4-metric; this is called a Eulerian frame ${ }^{56}$. An Eulerian frame can also be chosen in such a way that $\mathrm{L}_{\boldsymbol{U}} \sqrt{g}=0$, a condition called 'maximal slicing' ${ }^{57}$ [also Lichnerowicz (1944)].

The integral over a 3-surface is related to the number of tubes that intersect it.

We can take the tubes associated with $M$ as small as we please: in the limit they represent the trajectories in spacetime of 'particles' of the continuum.

\section{Cinstein: matter as geometry}

One of Einstein's goals was to represent matter as a manifestation of the gravitational field ${ }^{58}$. He tried to achieve this by representing matter particles as singularities of the field ${ }^{59}$, but this programme wasn't very successful.

$5 \mathbf{5 5}$ Misner et al. 1973 ch. $15 . \quad 56$ Smarr et al. 1978; 1980. $\quad{ }^{\mathbf{5} 7}$ Smarr et al. 1978 § III.B. $\quad{ }^{\mathbf{5 8}}$ see Havas et al. 1962; Havas 1967. ${ }^{\mathbf{5 9}}$ Einstein et al. 1938. 
The present note explores the idea of realizing Einstein's wish in a slightly different fashion: by interpreting mass, energy, and their fluxes as manifestations of the gravitational field. These manifestations depend on the temperature. Moreover, the gravitational field can interact with itself in a variety of ways, also dependent on the temperature; these ways are expressed in the stress. This idea appears when we try to combine Newtonian continuum thermomechanics and general relativity.

To explore this idea I first give in $\S \mathrm{D}$ a summary of the aim of Newtonian continuum thermomechanics and of its basic equations, stressing the importance of constitutive equations. It is necessary to discuss the 'aim' because, independently of the different invariance groups and geometrical structures of Newtonian mechanics and general relativity, the two theories seem to have different perspectives regarding the modelling of matter and fields. Newtonian continuum thermomechanics is a theory of crystal conceptual and methodological clarity, thanks to its 300 years of history. Some of its concepts and methods may be brought into general relativity and offer new interpretations of the latter.

I apologize for $\S \mathrm{D}$, which is actually very confusing, because I tried to keep it short and to give the broadest possible view at the same time. To correct this I give a summary of its key points at the end of it.

Then in § 8 I give a summary of the ' $3+1$ ' (or 'Arnowitt-Deser-Misner', or 'initial-value') formulation of the Einstein equations, showing in particular how the energy-momentum-stress balance equations arise from them. I then merge the $3+1$ formulation with some of the continuummechanical concepts previously summarized, also discussing some points that aren't clear to me.

I conclude with a $3+1$ spacetime presentation of Newtonian continuum mechanics, slightly different from Newton-Cartan theory. This presentation shows that the Newtonian geometrical structure is not so different from the general-relativistic one, and also reveals that the interpretation of some core concepts becomes actually foggier in general relativity.

This note excludes quantum theory and particle physics; concepts like particle, point-mass, baryon number never enter the discussion - I'm not familiar with baryon conservation. Our point of view and primitives are based on mass, force, energy, equations of balance and motion, and similar. Lagrangeans, Hamiltonians, actions, extremum principles, and similar notions are also avoided because I don't like them. 


\section{Continuum thermomechanics}

\section{D.1 Spacetime formulation}

The kinematic structures of Newtonian and general-relativistic mechanics are very similar if not identical. We start from a four-dimensional differential manifold representing spacetime. No metric or connection are given yet. On this manifold we choose a slicing determined by a function $t$, or equivalently by an exact 1 -form $\mathrm{d} t$.

We assume that each 3-surface, or slice, of this slicing is equipped with a 3-metric $\boldsymbol{g}_{t}$ and a metric-compatible connection $\nabla$.

In Newtonian mechanics we postulate the existence of an 'absolute time', represented by a particular slicing. Its 3-metrics are postulated to be Euclidean, and therefore their connections are flat. These postulates, however, are of a dynamic, not kinematic, nature; they are related to inertial and gravitational forces. Nothing forbids us to use a different slicing to describe the flow of matter.

Although each slice has a connection, no connection is defined on the whole spacetime. This means that there is no canonical correspondence between points on two different slices: we cannot say 'this place, now, is the same as that place, yesterday'. Such a correspondence is made via a vector field $U$ such that

$$
u \cdot \mathrm{d} t=1 .
$$

The flow of this vector field puts points on different slices into mutual correspondence. It's variously called an 'observer', or a field of observers, or a frame. In Newtonian mechanics we can choose frames that preserve the Euclidean metric of the slices: $\mathrm{L}_{\boldsymbol{u}} \boldsymbol{g}=0$, called rigid frames. In general relativity it is generally impossible to choose a vector field this way, but we can choose one normal to the slices with respect to a spacetime 4-metric; this is called a Eulerian frame ${ }^{60}$. An Eulerian frame can also be chosen in such a way that $\mathrm{L}_{u} \sqrt{g}=0$, a condition called 'maximal slicing' ${ }^{61}$ [also Lichnerowicz (1944)].

The tensors $\mathrm{d} t \otimes U$ and $\mathbf{I}-\mathrm{d} t \otimes U$ induce a projective decomposition of any geometric object into parts parallel to the vector field $U$, called timelike, and to parts parallel the slices of the slicing ${ }^{62}$, called spacelike. These projections do not depend on the metric or the connection.

$\overline{\mathbf{6 0}}$ Smarr et al. 1978; 1980. $\quad \mathbf{6 1}^{\mathbf{6}}$ Smarr et al. 1978 § III.B. $\quad{ }^{\mathbf{6 2}}$ Hehl et al. 2003 § B.1.4. 
The geometric structure just sketched is common to Newtonian mechanics and general relativity. In particular, also Newtonian mechanics has a 'lapse' and 'shift' in its equations, but they are chosen so as to make the gravito-inertial force assume the simplest possible form.

\section{D.2 Energy-force}

Mystery still surrounds the concepts of force and energy in Newtonian and general-relativistic mechanics and their differences in the two theories. In both theories the energy-force seems to be a covector-valued object, because it is meant to be integrated along a segment of line of matter flow $u$ to yield the work done by force on matter along that segment.

In Newtonian mechanics, force is traditionally an object that also has to be integrated on a closed 2-surface or a 3-volume to give the total force acting on matter within that volume. Forces $\boldsymbol{\tau}$ to be integrated over 2 -surfaces are called stresses; forces $f$ to be integrated over 3-volumes are called body forces. Such integration of a covector-valued object would require a flat connection: integrating means parallel-transporting the force covectors to a common point to sum them; but the result would depend on the path along which they are transported if the connection weren't flat ${ }^{63}$. The balance of force, however, stating that the total force on every 3-volume vanishes, can be expressed locally using a divergence, and this can be generalized to non-flat connections.

Very important is the gravito-inertial body force exerted on matter at some spacetime point, defined by

$$
f^{\star}:=N \gamma-U^{\star} \cdot \mathrm{L}_{U^{\star}} \boldsymbol{M} \cdot \mathbf{g}^{-1}
$$

and discussed in the previous section. Its definition in Newtonian mechanics requires a specific slicing, whose slices have Euclidean metrics $\boldsymbol{g}$ and a flat connection, and inertial and gravitational fields $\boldsymbol{U}^{\star}, \boldsymbol{\gamma}$. But besides this fact it can be treated similarly in both Newtonian and general-relativistic mechanics.

Most texts say that the 'time derivative of momentum', $m a$, is an effect of force. They also call 'inertial forces' some expressions appearing in non-inertial frames, and often say that these are 'fictitious'. But the spacetime covariant perspective shows that the quantity $-m a$, where $a$ is the acceleration with respect to the fixed stars has to be considered

63 Truesdell et al. 1960 § 238 . 
as a force. This force is the internal pressure we feel whenever we accelerate with respect to the fixed stars. It's rightly called inertial force. The gravitational force is given by the familiar expression $-m \gamma$, where $\gamma$ is the gravitational field.

In Newtonian mechanics the combined gravito-inertial force $f^{\star}$ depends locally on three quantities:

(1) the mass form $\boldsymbol{M}$,

(2) a particular frame $U^{\star}$, called the fixed-stars frame,

(3) the 3-metric $\mathbf{g}$.

Its exact expression $f^{\star}\left(\boldsymbol{M}, \boldsymbol{U}^{\star}, \boldsymbol{g}\right)$ in terms of these quantities is not important right now. It is something like $-N\left(\mathrm{~L}_{U^{\star} \boldsymbol{v}}+\gamma\right)$, where $v$ is a velocity covector obtained with the help of the metric $\mathbf{g}$ from the mass flux $\boldsymbol{U}^{\star} \cdot \boldsymbol{M}$. The gravitational force $\gamma$ is in turn determined locally by the mass 3-form $M$ via d $\gamma=-4 \pi G N$ - no metric or connection required. The fixed-stars frame $U^{\star}$ can also be thought as determined, non-locally, by the mass form $\boldsymbol{M}$ and the 3-metric $\boldsymbol{g}$, or possibly just the 3-connection. I discuss this in $\S^{* *}$; it is a form of Mach's principle.

The difference between Newtonian and general-relativistic mechanics is only in the dependence just explained, which is replaced by a local dependence in general relativity.

The fourth component of the gravito-inertial force is the change in kinetic and gravitational potential energies. The fact that kinetic energy is just the work per unit time done by the inertial force is clear from the relation $\partial_{t}\left(\frac{1}{2} m v^{2}\right)=\left(m \partial_{t} v\right) \cdot v$.

The discussion just given about the inertial force is very imprecise, but this lack of precision does not affect the discussion about the Einstein equations to be made later.

The second and third fundamental balances of continuum mechanics are those of force and energy, which says that the total energy-force on matter vanishes:

$$
f+\nabla \cdot T=0 .
$$

The gravito-inertial force is counted within the total body force $f$, and in a rigid frame with adapted coordinates it gives the familiar 'time 
derivative of momentum' term. In a rigid frame with adapted coordinates the above balances become

$$
\begin{aligned}
& \partial_{t} \epsilon=\nabla \cdot \boldsymbol{q}+h, \\
& \partial_{t} \boldsymbol{q}=\nabla \cdot \tau+\boldsymbol{b},
\end{aligned}
$$

with $\partial_{t}=\mathrm{L}_{\boldsymbol{u}}$. The heat flux $\boldsymbol{q}$ and the momentum $\boldsymbol{q}$ are separate entities in Newtonian mechanics, but in general relativity their distinction is not clear-cut: 'heat carries momentum' and 'momentum carries energy'64.

\section{D.3 Torque}

The concept of torque and its balance would require a rather long discussion. For the present note it is sufficient to remember that its balance traditionally leads to a symmetric stress tensor $\boldsymbol{\tau}$.

\section{D.4 Remaining conceptual problems}

We've seen that mass has a beautiful, conceptually intuitive, and metricfree geometric representation independent of the Newtonian or generalrelativistic setting. The appropriate geometric representation of force, energy, and their balance is still unclear and discussed today.

We could conjecture that metric and connection are only related to the definition of the gravito-inertial force, and the other kinds of forces do not need such structure. In that case the equation of energy-force balance (45) is unsatisfactory because it involves a covariant derivative and therefore a connection.

Relativity theory seems to suggest that we can speak about 'surface forces' in a generalized sense, where spacelike surfaces can also be considered, and the gravito-inertial force would be such a 'spacelike surface force'. The integration of such forces is still problematic, however, because spacetime is not flat. A way out of this could be to consider force - a covector - to be something that must first be contracted with a vector field - yielding a scalar-valued form - and then integrated. This vector field could be a field of 'virtual displacements', its contraction with the force yielding 'virtual work'; this is indeed another way to conceive force in Newtonian mechanics ${ }^{65}$. Some studies ${ }^{66}$ suggest that stress could be

$\mathbf{6 4}$ Eckart 1940b. $\quad \mathbf{6 5}$ Truesdell et al. 1960 § 238. $\quad \mathbf{6 6}$ Segev 2002; Hehl et al. 2003; Segev 1986; Segev et al. 1999; Segev 2000a,b. 
considered as a map transforming a 3-form like matter or charge into an energy 3 -form - thus with $4 \times 4$ real components, as required. This transformation would be metric-free.

Finally, an opposite conjecture is that force and energy could just be manifestations of the metric.

\section{D.5 Summary}

I imagine that this section on Newtonian mechanics was poorly written and very confusing. Unfortunately I haven't managed to find a good balance between shortness and broadness. I summarize here its 'takehome messages' necessary for the next section:

- Mass and its balance rely only on the differential-geometric structure. They are the same in Newtonian and general-relativistic mechanics.

- The kinematic of mass relies on the concepts of slicing and frame, chosen arbitrarily, without metrics or connections. It is the same in Newtonian and general-relativistic mechanics.

- Force and energy, and therefore the dynamic of mass, rely on the introduction of metrics and connections. They do so in similarl ways in Newtonian and general-relativistic mechanics.

- An important difference between Newtonian and generalrelativistic mechanics is in the form of the gravito-inertial force and its dependence on mass, metric, and a special slicing.

- The independent fields in Newtonian mechanics are mass 3-density, mass flux, temperature, 3-geometry. The energy-force tensor is given as a functional of them in a constitutive equation, which depends on the kind of matter considered. Mass balance, energy-force balance, and the constitutive equation yield a well-posed system of equations for the evolution of the independent fields.

\section{D.6 Doubts and remarks}

I believe that some points in the most common treatments of Newtonian and general-relativistic mechanics are an obstacle to a clearer understanding of the relationships between geometry, matter, and gravitation:

- The metric tensor is nonchalantly spread everywhere in the mathematical formulae: to raise or lower indices, introduce volume elements, and 
similar operations. But many physical quantities and their balance equations can actually be fully expressed in differential-geometrical, non-metric terms. This was shown, for example, by Kottler ${ }^{67}$ and van Dantzig ${ }^{68}$.

Mass density, mass flux, and the balance of mass are one example, as we saw in $\S 4$. Most literature instead ${ }^{69}$ writes the balance of mass introducing a metric volume element $\sqrt{|g|^{70}}$, but the metric should play no role here.

- The metric-compatible connection is also nonchalantly introduced everywhere from the start. Since metric and connection represent the gravito-inertial force, their introduction represents the appearance of dynamics before the kinematic is completed.

Studies by Segev ${ }^{71}$ indicate that the energy-momentum-stress tensor might also be defined in metric-free terms; compare the balance of momentum rewritten with the metric volume element in Smarr et al. ${ }^{72}$.

\section{Bibliography}

('de $X^{\prime}$ is listed under $\mathrm{D}$, 'van $X^{\prime}$ under $\mathrm{V}$, and so on, regardless of national conventions.) Alcubierre, M. (2008): Introduction to $3+1$ Numerical Relativity. (Oxford University Press, Oxford).

Alderman, J. L., Bergmann, O. (1970): Geometrical derivation of the conservation laws. J. Math. Phys. 11 ${ }^{5}, 1639-1644$.

Aldrovandi, R., Pereira, J. G. (2013): Teleparallel Gravity: An Introduction. (Springer, Dordrecht). Previously preprinted as http://www.ift. unesp.br/users/jpereira/te le.pdf.

Anile, A. M., Choquet-Bruhat, Y., eds. (1989): Relativistic Fluid Dynamics. (Springer, Berlin). Arcos, H. I., Pereirau, J. G. (2004): Torsion gravity: a reappraisal. Int. J. Mod. Phys. D 13 ${ }^{10}$, 2193-2240.

Ashtekar, A., Petkov, V., eds. (2014): Springer Handbook of Spacetime. (Springer, Berlin).

Bamberg, P., Sternberg, S. (1990): A course in mathematics for students of physics: 1. (Cambridge University Press, Cambridge). First publ. 1988.

Baumgarte, T. W., Shapiro, S. L. (2010): Numerical Relativity: Solving Einstein's Equations on the Computer. (Cambridge University Press, Cambridge).

Bernoulli, J. (1703): Démonstration générale du centre de balancement $\mathcal{E}$ d'oscillation, tirée de la nature du levier. Mémoires de mathématique et de physique tirés des registres de l'Académie Royale des Sciences 1703, 78-84.

$\mathbf{6 7}$ Kottler 1922a,b. $\mathbf{6 8}$ Van Dantzig 1934c,a,d,b,e; 1937; Schouten et al. 1940; van Dantzig 1954. ${ }^{69}$ e.g., Smarr et al. 1980 p. $171 . \quad 70$ cf. Choquet-Bruhat et al. 1996 § V.B.4, pp. 317-318. 71 Segev 2002. $\quad 72$ Smarr et al. 1980 p. 171. 
Bertotti, B., de Felice, F., Pascolini, A., eds. (1984): General Relativity and Gravitation: Invited Papers and Discussion Reports of the 10th International Conference on General Relativity and Gravitation, Padua, July 3-8, 1983. (Reidel, Dordrecht).

Bossavit, A. (1991): Differential Geometry: for the student of numerical methods in Electromagnetism. https://Www.researchgate.net/publication/200018385_Differential_Ge ometry_for_the_student_of_numerical_methods_in_Electromagnetism.

- (1998): On the geometry of electromagnetism. J. Japan Soc. Appl. Electromagn. \& Mech. 6, 17-28, 114-123, 233-240, 318-326. Corrected, combined version at https : / Www . resea rchgate.net/publication/254470625_0n_the_geometry_of_electromagnetism.

- (2001): On the notion of anisotropy of constitutive laws: some implications of the "Hodge implies metric" result. COMPEL 201 $233-239$.

Bressan, A. (1964): Una teoria di relatività generale includente, oltre all'elettromagnetismo e alla termodinamica, le equazioni costitutive dei materiali ereditari. Sistemazione assiomatica. Rend. Sem. Mat. Univ. Padova 34, 74-109.

Bunge, M., ed. (1967): Delaware Seminar in the Foundations of Physics. (Springer, Berlin).

Burke, W. L. (1980): Spacetime, Geometry, Cosmology. (University Science Books, Mill Valley, USA).

- (1983): Manifestly parity invariant electromagnetic theory and twisted tensors. J. Math. Phys. $24^{1}, 65-69$.

- (1987): Applied Differential Geometry, reprint. (Cambridge University Press, Cambridge). First publ. 1985.

- (1995): Div, Grad, Curl Are Dead. http://people.ucsc.edu/ rmont/papers/Burke_Di vGradCurl.pdf; 'preliminary draft II'. See also http://www. ucolick. org/ burke/.

Cai, Y.-F., Capozziello, S., De Laurentis, M., Saridakis, E. N. (2016): $f(T)$ teleparallel gravity and cosmology. Rep. Prog. Phys. 794, 106901.

Carter, B., Chachoua, E., Chamel, N. (2006): Covariant Newtonian and relativistic dynamics of (magneto)-elastic solid model for neutron star crust. Gen. Relat. Gravit. 38 ${ }^{1}, 83-119$.

Carter, B. (1973): Elastic perturbation theory in general relativity and a variation principle for a rotating solid star. Commun. Math. Phys. $\mathbf{3 0}^{4}, 261-286$.

Carter, B., Quintana, H. (1972): Foundations of general relativistic high-pressure elasticity theory. Proc. R. Soc. Lond. A $331^{1584}, 57-83$.

Choquet-Bruhat, Y., ed. (1974): Ondes et radiations gravitationnelles. (Centre National de la Recherche Scientifique, Paris).

- (2009): General Relativity and Einstein's Equations. (Oxford University Press, Oxford).

Choquet-Bruhat, Y., DeWitt-Morette, C., Dillard-Bleick, M. (1996): Analysis, Manifolds and Physics. Part I: Basics, rev. ed. (Elsevier, Amsterdam). First publ. 1977.

de Andrade, V. C., Guillen, L. C. T., Pereira, J. G. (2000): Teleparallel gravity: an overview. arXiv: gr-qc/0011087.

Disconzi, M. M. (2014): On the well-posedness of relativistic viscous fluids. Nonlinearity $27^{8}$, 1915-1935.

Disconzi, M. M., Kephart, T. W., Scherrer, R. J. (2017): On a viable first-order formulation of relativistic viscous fluids and its applications to cosmology. Int. J. Mod. Phys. D 26 ${ }^{13}$, 1750146.

Eckart, C. (1940a): The thermodynamics of irreversible processes. II. Fluid mixtures. Phys. Rev. $58^{3,10}, 269-275,924$.

- (1940b): The thermodynamics of irreversible processes. III. Relativistic theory of the simple fluid. Phys. Rev. 58 ${ }^{10}, 919-924$. 
Eddington, A. S. (1930): The Mathematical Theory of Relativity, 2nd ed. (Cambridge University Press, Cambridge). First publ. 1923.

Einstein, A., Infeld, L., Hoffmann, B. (1938): The gravitational equations and the problem of motion. Ann. Math. 39 ${ }^{1}, 65-100$.

Ericksen, J. L. (2007): On formulating and assessing continuum theories of electromagnetic fields in elastic materials. J. Elasticity $87^{2-3}, 95-108$.

Flügge, S., ed. (1960): Handbuch der Physik: Band III/1: Prinzipien der klassischen Mechanik und Feldtheorie [Encyclopedia of Physics: Vol. III/1: Principles of Classical Mechanics and Field Theory]. (Springer, Berlin).

Frankel, T. (2012): The Geometry of Physics: An Introduction, 3rd ed. (Cambridge University Press, Cambridge). First publ. 1997.

Frittelli, S. (1997): Note on the propagation of the constraints in standard $3+1$ general relativity. Phys. Rev. D 55 ${ }^{10}, 5992-5996$. http : / /www. andrew. cmu . edu/user/rgomez/preprints /prd55.5992.1997.pdf.

Geroch, R. (2001): On hyperbolic "theories" of relativistic dissipative fluids. arXiv:grqc/0103112.

Geroch, R., Jang, P. S. (1975): Motion of a body in general relativity. J. Math. Phys. 16 ${ }^{1}, 65-67$.

Geroch, R., Lindblom, L. (1991): Causal theories of dissipative relativistic fluids. Ann. of Phys. 207²,394-416. http://Www.ccom.ucsd.edu/ lindblom/Publications/45_AnnPhys.2 07. $394 . \mathrm{pdf}$.

Gourgoulhon, É. (2012): 3+1 Formalism in General Relativity: Bases of Numerical Relativity. (Springer, Heidelberg). First publ. 2007 as arXiv : gr-qc/0703035.

Grot, R. A., Eringen, A. C. (1966a): Relativistic continuum mechanics: Part I-Mechanics and thermodynamics. Int. J. Engng Sci. $4^{6}, 611-638,664$. See also Grot, Eringen (1966b).

- (1966b): Relativistic continuum mechanics: Part II - Electromagnetic interactions with matter. Int. J. Engng Sci. $4^{6}, 639-670$. See also Grot, Eringen (1966a).

Havas, P. (1967): Foundation problems in general relativity. In: Bunge (1967), ch. 8, 124-148.

Havas, P., Goldberg, J. N. (1962): Lorentz-invariant equations of motion of point masses in the general theory of relativity. Phys. Rev. 128 ${ }^{1}, 398-414$.

Hehl, F. W., Obukhov, Y. N. (2000): A gentle introduction to the foundations of classical electrodynamics: the meaning of the excitations $(\mathcal{D}, \mathcal{H})$ and the field strengths $(E, B)$. arXiv: physics/0005084.

- (2003): Foundations of Classical Electrodynamics: Charge, Flux, and Metric. (Birkhäuser, Boston).

Hehl, F. W., Obukhov, Y. N., Rubilar, G. F. (1999): Classical electrodynamics: a tutorial on its foundations. arXiv: physics/9907046.

Henkin, L., Suppes, P., Tarski, A., eds. (1959): The Axiomatic Method: With Special Reference to Geometry and Physics. (North-Holland, Amsterdam). http://www . archive . org/det ails/axiomaticmethod031862mbp.

Herrmann, H. J., Muschik, W., Rückner, G. (2000): Constitutive theory in general relativity: basic fields, state spaces and the principle of minimal coupling. Rend. Sem. Mat. Univ. Pol. Torino $58^{2}, 133-139$.

Hiscock, W. A., Lindblom, L. (1985): Generic instabilities in first-order dissipative relativistic fluid theories. Phys. Rev. D 31 ${ }^{4}, 725-733$.

ISO (International Organization for Standardization) (2009a): ISO 80000-1:2009: Quantities and units 1: General. International Organization for Standardization.

- (2009b): ISO 80000-9:2009: Quantities and units 9: Physical chemistry and molecular physics. International Organization for Standardization. 
JCGM (Joint Committee for Guides in Metrology) (2012): JCGM 200:2012: International vocabulary of metrology - Basic and general concepts and associated terms (VIM), 3rd ed. Joint Committee for Guides in Metrology (JCGM), BIPM, IEC, IFCC, ILAC, ISO, IUPAC, IUPAP, OIML. https : //www . bipm .org/en/publications/guides/vim. html. First publ. 1997.

Kanso, E., Arroyo, M., Tong, Y., Yavari, A., Marsden, J. G., Desbrun, M. (2007): On the geometric character of stress in continuum mechanics. Z. Angew. Math. Phys. 58 $\mathbf{8}^{5}, 843-856$. http://www. geometry.caltech.edu/pubs.html.

Kottler, F. (1922a): Newton'sches Gesetz und Metrik. Sitzungsber. Akad. Wiss. Wien, Math.Naturw. Klasse, Abt. IIa 1312 ${ }^{2}, 1-14$.

- (1922b): Maxwell'sche Gleichungen und Metrik. Sitzungsber. Akad. Wiss. Wien, Math.Naturw. Klasse, Abt. IIa 131² $119-146$.

Landau, L. D., Lifshitz [Lifšic], E. M. (1994): The Classical Theory of Fields, fourth English ed. (Butterworth-Heinemann, Oxford). Transl. from the 1987 seventh Russian edition by Morton Hamermesh. First publ. 1939.

Lianis, G. (1973): The general form of constitutive equations in relativistic physics. Nuovo Cimento B 14 ${ }^{1}, 57-103$.

Marsden, J. E., Hughes, T. J. R. (1994): Mathematical Foundations of Elasticity, unabridged corr. republ. (Dover, New York). http://resolver. caltech. edu/CaltechB00K: 1983 . 002 . First publ. 1983.

Maugin, G. A. (1971a): Magnetized deformable media in general relativity. Ann. Inst. Henri Poincaré (A) $15^{4}, 275-302$.

- (1971b): Un modèle viscoélastique en relativité générale. C. R. Acad. Sc. Paris Série A 272, 1482-1484.

- (1973): Harmonic oscillations of elastic continua and detection of gravitational waves. Gen. Relat. Gravit. $4^{3}, 241-272$.

- (1974a): The principle of material frame indifference in general relativity and the formulation of constitutive equations for relativistic continuous matter. In: Choquet-Bruhat (1974), 331-338.

- (1974b): Constitutive equations for heat conduction in general relativity. J. Phys. A $7^{4}$, 465-484.

- (1978a): On the covariant equations of the relativistic electrodynamics of continua. I. General equations. J. Math. Phys. 195, $1198-1205$.

- (1978b): Elasticity and electro-magneto-elasticity of general-relativistic systems. Gen. Relat. Gravit. $9^{6}, 541-549$.

- (1978c): On the covariant equations of the relativistic electrodynamics of continua. II. Fluids. J. Math. Phys. 195 $1206-1211$.

- (1978d): On the covariant equations of the relativistic electrodynamics of continua. III. Elastic solids. J. Math. Phys. 195, 1212-1219.

- (1978e): On the covariant equations of the relativistic electrodynamics of continua. IV. Media with spin. J. Math. Phys. $19^{5}, 1220-1226$.

Maugin, G. A., Eringen, A. C. (1972a): Relativistic continua with directors. J. Math. Phys. 13 ${ }^{11}$, 1788-1797.

- (1972b): Polarized elastic materials with electronic spin - a relativistic approach. J. Math. Phys. 13 ${ }^{11}, 1777-1788$.

Misner, C. W., Thorne, K. S., Wheeler, J. A. (1973): Gravitation, repr. (W. H. Freeman and Company, New York). https://archive.org/details/Gravitation_201803. 
Noll, W. (1959): The foundations of classical mechanics in the light of recent advances in continuum mechanics. In: Henkin, Suppes, Tarski (1959), 266-281. Repr. in Noll (1974), pp. 32-47.

- (1963): La m'ecanique classique, basée sur un axiome d'objectivité. In: La méthode axiomatique dans les mécaniques classiques et nouvelles (Gauthier-Villars, Paris), 47-56. Repr. in Noll (1974), pp. 135-144.

- (1973): Lectures on the foundations of continuum mechanics and thermodynamics. Arch. Rational Mech. Anal. 52 ${ }^{1}, 62-92$.

- (1974): The Foundations of Mechanics and Thermodynamics: Selected Papers. (Springer). With a preface by C. Truesdell.

Page, L. (1958): Introduction to Theoretical Physics, third ed., third printing. (Van Nostrand, Princeton, USA). First publ. 1928.

Pauli, W. (1958): Theory of Relativity. (Pergamon, London). Transl. by G. Field; with supplementary notes by W. Pauli. First publ. in German 1921.

Pereira, J. G. (2014): Teleparallelism: a new insight into gravity. In: Ashtekar, Petkov (2014), ch. 11, 197-212.

Pimentel, O. M., Lora-Clavijo, F. D., González, G. A. (2016): The energy-momentum tensor for a dissipative fluid in general relativity. Gen. Relat. Gravit. $48^{10}, 124$.

Rezzolla, L., Zanotti, O. (2013): Relativistic Hydrodynamics. (Oxford University Press, Oxford).

Samohýl, I. (1987): Thermodynamics of Irreversible Processes in Fluid Mixtures (Approached by Rational Thermodynamics). (Teubner, Leipzig). Partially rewritten 2014 as Samohýl, Pekař (2014).

Samohýl, I., Pekař, M. (2014): The Thermodynamics of Linear Fluids and Fluid Mixtures. (Springer, Cham). First published 1987 as Samohýl (1987).

Schilpp, P. A., ed. (1970): Albert Einstein: Philosopher-Scientist, 3rd ed. (MJF Books, New York). First publ. 1949.

Schouten, J. A. (1951): Tensor Analysis for Physicists. (Oxford University Press, Oxford).

Schouten, J. A., van Dantzig, D. (1940): On ordinary quantities and W-quantities. Classification and geometrical applications. Compos. Math. 7, 447-473.

Segev, R. (1986): Forces and the existence of stresses in invariant continuum mechanics. J. Math. Phys. 27 ${ }^{1}, 163-170$. http://www. bgu .ac.il/ rsegev/Papers/JMP86S.pdf.

- (2000a): The geometry of Cauchy's fluxes. Arch. Rational Mech. Anal. 154²,183-198. htt p://www.bgu.ac.il/ rsegev/Papers/TheGeometryofCauchyFluxes.pdf.

- (2000b): Notes on stresses for manifolds. Rend. Sem. Mat. Univ. Pol. Torino 58 ${ }^{2}, 199-206$.

- (2001): A correction of an inconsistency in my paper "Cauchy's theorem on manifolds". J. Elasticity $\mathbf{6 3}^{1}, 55-59$. Ssee Segev, Rodnay (1999).

- (2002): Metric-independent analysis of the stress-energy tensor. J. Math. Phys. $43^{6}, 3220$ 3231. http://www. bgu.ac.il/ rsegev/Papers/MetricIndependent.pdf.

Segev, R., Rodnay, G. (1999): Cauchy's theorem on manifolds. J. Elasticity $56^{2}$, 129-144. h ttp : //www. bgu . ac.il/ rsegev/Papers/CauchyTheoremonManifolds. pdf. See also erratum Segev (2001).

- (2000): The divergence of stress and the principle of virtual power on manifolds. Technische Mechanik 20², 129-136. http://www. bgu.ac.il/ rsegev/Papers/DivergenceofStre ssesPrinciple.pdf.

- (2012): The co-divergence of vector valued currents. Discrete and Continuous Dynamical Systems B 17², 687-698.

Smarr, L. L., ed. (1979): Sources of Gravitational Radiation. (Cambridge University Press, Cambridge). 
Smarr, L., Taubes, C., Wilson, J. R. (1980): General relativistic hydrodynamics: the comoving, Eulerian, and velocity potential formalisms. In: Tipler (1980), ch. 11, 157-183.

Smarr, L., York Jr., J. W. (1978): Kinematical conditions in the construction of spacetime. Phys. Rev. D 17 ${ }^{10}, 2529-2551$.

Tipler, F. J., ed. (1980): Essays in General Relativity: A Festschrift for Abraham Taub. (Academic Press, New York).

Truesdell III, C. A. (1968): Essays in the History of Mechanics. (Springer, Berlin).

- (1984): Rational Thermodynamics, 2nd ed. (Springer, New York). First publ. 1969.

- (1991): A First Course in Rational Continuum Mechanics. Vol. 1: General Concepts, 2nd ed. (Academic Press, New York). First publ. 1977.

Truesdell III, C. A., Toupin, R. A. (1960): The Classical Field Theories. In: Flügge (1960), I-VII, 226-902. With an appendix on invariants by Jerald LaVerne Ericksen.

van Dantzig, D. (1934a): Electromagnetism, independent of metrical geometry. 1. The foundations. Proc. Acad. Sci. Amsterdam (Proc. of the Section of Sciences Koninklijke Nederlandse Akademie van Wetenschappen) 37, 521-525. See also van Dantzig (1934d,b,e; 1936).

- (1934b): Electromagnetism, independent of metrical geometry. 3. Mass and motion. Proc. Acad. Sci. Amsterdam (Proc. of the Section of Sciences Koninklijke Nederlandse Akademie van Wetenschappen) 37, 643-652. See also van Dantzig (1934a,d,e; 1936).

- (1934c): The fundamentals equations of electromagnetism, independent of metrical geometry. Proc. Cambridge Philos. Soc. $\mathbf{3 0}^{4}, \mathbf{4 2 1 - 4 2 7 .}$

- (1934d): Electromagnetism, independent of metrical geometry. 2. Variational principles and further generalisation of the theory. Proc. Acad. Sci. Amsterdam (Proc. of the Section of Sciences Koninklijke Nederlandse Akademie van Wetenschappen) 37, 526-531. See also van Dantzig (1934a,b,e; 1936).

- (1934e): Electromagnetism, independent of metrical geometry. 4. Momentum and energy; waves. Proc. Acad. Sci. Amsterdam (Proc. of the Section of Sciences Koninklijke Nederlandse Akademie van Wetenschappen) 37, 825-836. See also van Dantzig (1934a,d,b; 1936).

- (1936): Electromagnetism, independent of metrical geometry. 5. Quantum-theoretical commutability-relations for light-waves. Proc. Acad. Sci. Amsterdam (Proc. of the Section of Sciences Koninklijke Nederlandse Akademie van Wetenschappen) 39, 126-131. See also van Dantzig (1934a,d,b,e).

- (1937): Some possibilities of the future development of the notions of space and time. Erkenntnis $7^{1}, 142-146$.

- (1954): On the geometrical representation of elementary physical objects and the relations between geometry and physics. Nieuw Archief voor Wiskunde II, 73-89.

von Laue, M. (1949): Inertia and energy. In: Schilpp (1970), p. II.19, 501-533.

Weatherall, J. O. (2011): The motion of a body in Newtonian theories. J. Math. Phys. $\mathbf{5 2}^{3}, 032502$. http://jamesowenweatherall. com/publications/.

- (2017): Conservation, inertia, and spacetime geometry. Stud. Hist. Philos. Mod. Phys. ${ }^{* * *}$, **. http://jamesowenweatherall.com/publications/.

Wilson, J. R., Mathews, G. J. (2007): Relativistic Numerical Hydrodynamics, reprint. (Cambridge University Press, Cambridge). First publ. 2003.

York Jr., J. W. (1979): Kinematics and dynamics of general relativity. In: Smarr (1979), 83-126. 